\title{
Synthesis of Controllable and Normal Sublanguages for Discrete-Event Systems using a Coordinator
}

\author{
Jan Komenda ${ }^{\mathrm{a}}$, Tomáš Masopust ${ }^{\mathrm{a}, \mathrm{b}, *}$, Jan H. van Schuppen ${ }^{\mathrm{b}}$ \\ ${ }^{a}$ Institute of Mathematics, Czech Academy of Sciences, Žižkova 22, 61662 Brno, Czech Republic \\ ${ }^{b}$ CWI, P.O. Box 94079, 1090 GB Amsterdam, The Netherlands
}

\begin{abstract}
Synthesis of normal or controllable and normal sublanguages of global specification languages without computation of the global modular plant is a difficult problem. In this paper, these sublanguages are computed using a coordinator. We recall the notion of conditional controllability, introduce a notion of conditional normality, and prove necessary and sufficient conditions where such a computation is possible. Specifically, we show that conditionally controllable and conditionally normal languages computed by our method are controllable and normal with respect to the global plant. The optimality (supremality) of the resulting languages is also discussed.
\end{abstract}

Keywords: Discrete-event system, coordination control, coordinator, supervisory control, conditional controllability, conditional observability, conditional normality, supremal conditionally controllable and conditionally normal sublanguage.

2000 MSC: 93C65, 93A99, 93B50

\section{Introduction}

In supervisory control with partial observations, specification languages must be observable and controllable for a supervisor to exist so that the closed-loop system equals the specification [1]. This condition is often not satisfied and, moreover, a control requirement is most often a so called safety specification, that is, only the inclusion of the closed-loop language in the specification is required. In the case when the specification language is not controllable or observable, a controllable and observable sublanguage of the specification is considered. The synthesis of observable sublanguages is difficult, especially in the modular setting, where the plant is composed of local subsystems (automata) running in parallel so that the plant is formed as a synchronous product of local components.

Unfortunately, observability is not preserved under union, unlike controllability. Therefore, the supremal observable sublanguage does not always exist, and there are only maximal observable sublanguages, which are not unique in general. A slightly stronger notion, called normality, coincides with observability in the case when all controllable events are observable. Supremal normal sublanguages exist, but they are difficult to compute, especially in the modular framework. We have studied possibilities of local (modular) computations of supremal normal sublanguages in [2] for local specification languages and in [3] for global specification languages. However, the sufficient conditions for their local computation to equal the global computation are too restrictive.

In this paper, another approach is presented for synthesis of controllable and normal sublanguages in modular discrete-event systems. This approach is much less restrictive, but the optimality (supremality of the computed sublanguage) is only guaranteed under some additional conditions. It is based on the coordination control architecture, where a coordinator is added to the plant that takes care of the global part of the specification. Since only prefix-closed specifications are considered in this paper, it is not the coordinator automaton itself, but only its underlying event set that is the basis of our approach. In fact, coordinators themselves are useful for handling the blocking issues, while in

\footnotetext{
${ }^{*}$ Corresponding author. CWI, P.O. Box 94079, 1090 GB Amsterdam, The Netherlands, Tel. +31(0)205924122, Fax. +31(0)205924199

Email addresses: komenda@ipm.cz (Jan Komenda), masopust@math. cas. cz (Tomáš Masopust), J.H.van.Schuppen@cwi .nl (Jan H. van Schuppen)
} 
the prefix-closed case it is sufficient to choose a suitable event set containing the intersection of local event sets. The coordinator is then simply the global system projected to this coordinator event set so that the system composed with the coordinator equals the original modular plant. Moreover, the coordinator can be computed modularly because of the conditional independence property (amounting to distributivity of natural projections). The event set of the coordinator is chosen so that the specification language is conditional decomposable as defined in the paper. Our results are based on the notion of conditional controllability and conditional normality, and the computation of supremal conditionally controllable and conditionally normal sublanguages of the specification computed without building the global plant. It is shown that supremal conditionally controllable and conditionally normal sublanguages are controllable and normal with respect to the coordinated plant that equals the original plant as discussed above. Moreover, it is shown that, under some additional conditions, supremal conditionally controllable and conditionally normal, and supremal controllable and normal sublanguages coincide.

The paper is organized as described below. The next section recalls the basic results of supervisory control theory used further in the paper. In Section 3, basic notions that are needed for our approach are introduced. Section 4 contains further concepts on partially observed modular plants, in particular a necessary and sufficient condition for the specification to be exactly achievable using our coordination control scheme, called conditional observability. In Section 5, a stronger notion, called conditional normality, is presented. In Section 6, a procedure for the computation of supremal conditionally controllable and conditional normal sublanguages is proposed. Finally, a conclusion is given in Section 7.

\section{Preliminaries}

In this section, we briefly recall the elements of supervisory control theory needed in this paper. For more details, the reader is referred to $[1,4]$.

Discrete-event systems are modeled as deterministic generators that are finite-state machines with partial transition functions, i.e., a generator is a quintuple $G=\left(Q, E, f, q_{0}, Q_{m}\right)$, where $Q$ is a finite set of states, $E$ is the finite event set, $f: Q \times E \rightarrow Q$ is the partial transition function, $q_{0} \in Q$ is the initial state, and $Q_{m} \subseteq Q$ is the set of marked states. As usual, $f$ is extended to $f: Q \times E^{*} \rightarrow Q$. The behaviors of $G$ are defined in terms of languages. The language generated by $G$ is defined as $L(G)=\left\{s \in E^{*} \mid f\left(q_{0}, s\right) \in Q\right\}$, and the language marked by $G$ is defined as $L_{m}(G)=\left\{s \in E^{*} \mid f\left(q_{0}, s\right) \in Q_{m}\right\}$.

The prefix closure $\bar{L}$ of a language $L \subseteq E^{*}$ is the set of all prefixes of all its words, i.e., $\bar{L}=\left\{w \in E^{*} \mid \exists v \in\right.$ $E^{*}$ such that $\left.w v \in L\right\}$. A language $L$ is prefix-closed if $L=\bar{L}$.

Let $L$ be a prefix-closed language over an event set $E$ with the uncontrollable event set $E_{u} \subseteq E$. A language $K \subseteq E^{*}$ is controllable with respect to $L$ and $E_{u}$ if

$$
\bar{K} E_{u} \cap L \subseteq \bar{K} .
$$

A natural projection $P: E^{*} \rightarrow E_{o}^{*}$, for some $E_{o} \subseteq E$, is a homomorphism defined so that $P(a)=\varepsilon$, for $a \in E \backslash E_{o}$, and $P(a)=a$, for $a \in E_{o}$. The inverse image of $P$, denoted by $P^{-1}: E_{o}^{*} \rightarrow 2^{E^{*}}$, is defined as $P^{-1}(a)=\left\{s \in E^{*} \mid P(s)=\right.$ $a\}$. These definitions can naturally be extended to languages.

In what follows, given event sets $E_{i}, E_{j}, E_{k}$, we denote by $P_{k}^{i+j}$ the natural projection from $\left(E_{i} \cup E_{j}\right)^{*}$ to $E_{k}^{*}$, and by $P_{j \cap k}^{i}$ the natural projection from $E_{i}^{*}$ to $\left(E_{j} \cap E_{k}\right)^{*}$. In addition, we define $E_{i, o}=E_{i} \cap E_{o}, E_{i, u}=E_{i} \cap E_{u}$, etc.

Let $K$ and $M=\bar{M}$ be languages over an event set $E$. Let $E_{c} \subseteq E$ be the subset of controllable events, and let $E_{o} \subseteq E$ be the set of observable events with $P$ as the corresponding natural projection from $E^{*}$ to $E_{o}^{*}$. The specification language $K$ is said to be observable with respect to $M, E_{o}$, and $E_{c}$ if for all $s \in \bar{K}$ and for all $\sigma \in E_{c}$,

$$
(s \sigma \notin \bar{K}) \text { and }(s \sigma \in M) \Rightarrow P^{-1}[P(s)] \sigma \cap \bar{K}=\emptyset .
$$

Consider $M=\bar{M} \subseteq E^{*}$ and a natural projection $P: E^{*} \rightarrow E_{o}^{*}$. A language $K \subseteq M$ is said to be normal with respect to $M$ and $P$ if

$$
\bar{K}=P^{-1}[P(\bar{K})] \cap M .
$$

Note that it is known that normality implies observability [1]. 
A controlled generator is a structure $\left(G, E_{c}, P, \Gamma\right)$, where $G$ is a generator, $E_{c} \subseteq E$ is the set of controllable events, $E_{u}=E \backslash E_{c}$ is the set of uncontrollable events, $P: E^{*} \rightarrow E_{o}^{*}$ is the natural projection, and $\Gamma=\left\{\gamma \subseteq E \mid E_{u} \subseteq \gamma\right\}$ is the set of control patterns.

A supervisor for the controlled generator $\left(G, E_{c}, P, \Gamma\right)$ is a map $S: P(L(G)) \rightarrow \Gamma$.

A closed-loop system associated with the controlled generator $\left(G, E_{c}, P, \Gamma\right)$ and the supervisor $S$ is defined as the smallest language $L(S / G) \subseteq E^{*}$ which satisfies

1. $\varepsilon \in L(S / G)$,

2. if $s \in L(S / G)$, $s a \in L(G)$, and $a \in S(P(s))$, then $s a \in L(S / G)$.

In the automata framework where the supervisor is represented as an automaton, one can write $L(S / G)$ as $L(S) \| L(G)$.

Let $G$ be a generator over an event set $E$. Let $E_{u} \subseteq E$ be the set of uncontrollable events, $E_{c}=E \backslash E_{u}$ be the set of controllable events, and $E_{o} \subseteq E$ be the set of observable events. Given a prefix-closed specification language $K \subseteq L(G) \subseteq E^{*}$, the aim of supervisory control theory is to find a supervisor $S$ such that $L(S / G)=K$. It is known that such a supervisor exists if and only if $K$ is controllable with respect to $L(G)$ and $E_{u}$ and observable with respect to $L(G), E_{o}$, and $E_{c}$ [1]. However, as there are not, in general, supremal observable sublanguages, normality is used instead of observability. Thus, for specifications that are either not controllable or not observable, controllable and normal sublanguages are considered. In what follows, for prefix-closed languages $K \subseteq L \subseteq E^{*}, E_{u}, E_{o} \subseteq E$, and $Q: E^{*} \rightarrow E_{o}^{*}$, the notation sup $\mathrm{CN}\left(K, L, E_{u}, Q\right)$ is chosen for the supremal controllable and normal sublanguage of $K$ with respect to $L, E_{u}$, and $Q$. This supremal controllable and normal sublanguage always exists and equals the union of all controllable and normal sublanguages of $K$, see, e.g, [1]. A formula for calculating supremal controllable and normal sublanguages can be found in [5].

Below, modular discrete-event systems are considered. First, we recall that the synchronous product of languages $L_{1} \subseteq E_{1}^{*}$ and $L_{2} \subseteq E_{2}^{*}$ is defined by

$$
L_{1} \| L_{2}=P_{1}^{-1}\left(L_{1}\right) \cap P_{2}^{-1}\left(L_{2}\right) \subseteq E^{*},
$$

where $P_{i}: E^{*} \rightarrow E_{i}^{*}$, for $i=1,2$, are natural projections to local event sets. The synchronous product can also be defined for generators (the reader is referred to [1] for more details). In this case, for two generators $G_{1}$ and $G_{2}$, it is well known that $L\left(G_{1} \| G_{2}\right)=L\left(G_{1}\right) \| L\left(G_{2}\right)$ and $L_{m}\left(G_{1} \| G_{2}\right)=L_{m}\left(G_{1}\right) \| L_{m}\left(G_{2}\right)$.

Decentralized control is control of a monolithic system with two or more controllers each having its own observation channel. The observation event sets of the observation channels are incomparable in general. A modular or a distributed discrete-event system is the synchronous product of two or more modules or subsystems. In control of a distributed system each module or subsystem has its own observation channel and a supervisor or controller either with locally complete or locally partial observations. Control synthesis of a distributed discrete-event system then consists of synthesizing local supervisors, one for each subsystem. This is the main problem of the paper for which the coordinated control synthesis procedure is proposed. The global supervisor then consists of the synchronous product of local supervisors although that product is not computed in practice. In terms of behaviors, the optimal global control synthesis is represented by the closed-loop language $\sup \mathrm{CN}\left(K, L, E_{u}, Q\right)=\sup \mathrm{CN}\left(\left\|_{i=1}^{n} K_{i},\right\|_{i=1}^{n} L_{i}, E_{u}, Q\right)$.

Given a rational global specification language $K \subseteq E^{*}$, one can theoretically always compute its supremal controllable and normal sublanguage from which the optimal (least restrictive) supervisor can be built. Such a global control synthesis of a modular discrete-event system consists simply in computing the global plant and then the control synthesis is carried out as described above.

Decentralized control synthesis means that the specification language $K$ is replaced by $K_{i}=K \cap P_{i}^{-1}\left(L_{i}\right)$, and the synthesis is done similarly as for local specifications or using the notion of partial controllability [6]. However, the purely decentralized control synthesis is not always possible as the sufficient conditions under which it can be used are quite restrictive. Therefore, we have proposed the coordination control in [7] as a trade-off between the purely decentralized control synthesis, which is in some cases unrealistic, and the global control synthesis, which is naturally prohibitive for complexity reasons.

\section{Concepts}

Consider three generators $G_{1}, G_{2}$, and $G_{k}$. We call $G_{1}$ and $G_{2}$ conditionally independent generators given $G_{k}$ if there is no simultaneous move in both $G_{1}$ and $G_{2}$ without the coordinator $G_{k}$ being also involved, i.e.,

$$
E_{r}\left(G_{1} \| G_{2}\right) \cap E_{r}\left(G_{1}\right) \cap E_{r}\left(G_{2}\right) \subseteq E_{r}\left(G_{k}\right),
$$


where $E_{r}(G)$ is the set of all reachable symbols in $G$, i.e., symbols that appear in a word of the language $L(G)$. This concept can easily be extended to the case of three or more generators. The corresponding concept in terms of languages follows. Consider event sets $E_{1}, E_{2}, E_{k}$, and languages $L_{1} \subseteq E_{1}^{*}, L_{2} \subseteq E_{2}^{*}$, and $L_{k} \subseteq E_{k}^{*}$. The languages $L_{1}$ and $L_{2}$ are conditionally independent given $L_{k}$ if

$$
E_{r}\left(L_{1} \| L_{2}\right) \cap E_{1} \cap E_{2} \subseteq E_{k},
$$

where $E_{r}(L)$ denotes the set of all symbols occurring in words of $L$.

A language $K \subseteq\left(E_{1} \cup E_{2} \cup E_{k}\right)^{*}$ is called conditionally decomposable with respect to event sets $\left(E_{1}, E_{2}, E_{k}\right)$ if

$$
K=P_{1+k}(K)\left\|P_{2+k}(K)\right\| P_{k}(K) .
$$

Now, the problem studied in this paper is formulated.

Problem 1. Consider generators $G_{1}, G_{2}, G_{k}$ with event sets $E_{1}, E_{2}, E_{k}$, respectively, and a prefix-closed specification language $K \subseteq L\left(G_{1}\left\|G_{2}\right\| G_{k}\right)$. Assume that the coordinator $G_{k}$ makes the two generators $G_{1}$ and $G_{2}$ conditionally independent, and that the specification language $K$ is conditionally decomposable with respect to event sets $\left(E_{1}, E_{2}, E_{k}\right)$.

The overall control task is divided into local subtasks and the coordinator subtask [7]. The coordinator takes care of its "part" of the specification, namely $P_{k}(K)$, i.e., $L\left(S_{k} / G_{k}\right) \subseteq P_{k}(K)$. Similarly, supervisors $S_{1}$ and $S_{2}$ take care of their corresponding "parts" of the specification, namely $P_{i+k}(K)$, i.e., $L\left(S_{i} /\left[G_{i} \|\left(S_{k} / G_{k}\right)\right]\right) \subseteq P_{i+k}(K)$, for $i=1,2$.

Determine supervisors $S_{1}, S_{2}$, and $S_{k}$ for the respective generators so that the closed-loop system with the coordinator is such that

$$
L\left(S_{1} /\left[G_{1} \|\left(S_{k} / G_{k}\right)\right]\right)\left\|L\left(S_{2} /\left[G_{2} \|\left(S_{k} / G_{k}\right)\right]\right)\right\| L\left(S_{k} / G_{k}\right)=K
$$

In Section 6.1, we discuss the question of how to find a coordinator as well as we demonstrate the method suggested in this paper. However, note that efficient algorithms that are not discussed in the paper are still a part of our future research.

In what follows, the notion of conditional controllability plays a key role in the theory.

Definition 2. Consider the setting of Problem 1. Call the specification language $K \subseteq E^{*}$ conditionally controllable for generators $\left(G_{1}, G_{2}, G_{k}\right)$ and for the (uncontrollable) event subsets $\left(E_{1+k, u}, E_{2+k, u}, E_{k, u}\right)$ if

(i) The language $P_{k}(K) \subseteq E_{k}^{*}$ is controllable with respect to $G_{k}$ and $E_{k, u}$; equivalently,

$$
P_{k}(K) E_{k, u} \cap L\left(G_{k}\right) \subseteq P_{k}(K) .
$$

(ii.a) The language $P_{1+k}(K) \subseteq\left(E_{1} \cup E_{k}\right)^{*}$ is controllable with respect to $L\left(G_{1}\right)\left\|P_{k}(K)\right\| P_{k}^{2+k}\left(L\left(G_{2}\right) \| P_{k}(K)\right)$ and $E_{1+k, u}=$ $E_{u} \cap\left(E_{1} \cup E_{k}\right)$; equivalently,

$$
P_{1+k}(K) E_{1+k, u} \cap L\left(G_{1}\right)\left\|P_{k}(K)\right\| P_{k}^{2+k}\left(L\left(G_{2}\right) \| P_{k}(K)\right) \subseteq P_{1+k}(K) .
$$

(ii.b) The language $P_{2+k}(K) \subseteq\left(E_{2} \cup E_{k}\right)^{*}$ is controllable with respect to $L\left(G_{2}\right)\left\|P_{k}(K)\right\| P_{k}^{1+k}\left(L\left(G_{1}\right) \| P_{k}(K)\right)$ and $E_{2+k, u}$; equivalently,

$$
P_{2+k}(K) E_{2+k, u} \cap L\left(G_{2}\right)\left\|P_{k}(K)\right\| P_{k}^{1+k}\left(L\left(G_{1}\right) \| P_{k}(K)\right) \subseteq P_{2+k}(K) .
$$

\section{Control synthesis of conditionally observable and conditionally controllable languages}

In this section, we introduce a notion of conditional observability and prove that this condition along with conditional controllability are necessary and sufficient conditions for a specification language to be exactly achieved according to the setting of Problem 1.

Definition 3. Consider the setting of Problem 1. Call the specification language $K \subseteq E^{*}$ conditionally observable for generators $\left(G_{1}, G_{2}, G_{k}\right)$, controllable subsets $\left(E_{1+k, c}, E_{2+k, c}, E_{k, c}\right)$, and natural projections $\left(Q_{1+k}, Q_{2+k}, Q_{k}\right)$, where $Q_{i}: E_{i}^{*} \rightarrow E_{i, o}^{*}$, for $i=1+k, 2+k, k$, if 
(i) The language $P_{k}(K) \subseteq E_{k}^{*}$ is observable with respect to $L\left(G_{k}\right), E_{k, c}$, and $Q_{k}$; equivalently, for all $s \in P_{k}(K)$ and for all $\sigma \in E_{k, c}$,

$$
\left(s \sigma \notin P_{k}(K)\right) \text { and }\left(s \sigma \in L\left(G_{k}\right)\right) \Rightarrow Q_{k}^{-1}\left[Q_{k}(s)\right] \sigma \cap P_{k}(K)=\emptyset \text {. }
$$

(ii.a) The language $P_{1+k}(K) \subseteq\left(E_{1} \cup E_{k}\right)^{*}$ is observable with respect to $L\left(G_{1}\right)\left\|P_{k}(K)\right\| P_{k}^{2+k}\left(L\left(G_{2}\right) \| P_{k}(K)\right), E_{1+k, c}=$ $E_{c} \cap\left(E_{1} \cup E_{k}\right)$, and $Q_{1+k}$; equivalently, for all $s \in P_{1+k}(K)$ and for all $\sigma \in E_{1+k, c}$,

$$
\left(s \sigma \notin P_{1+k}(K)\right) \text { and }\left(s \sigma \in L\left(G_{1}\right)\left\|P_{k}(K)\right\| P_{k}^{2+k}\left(L\left(G_{2}\right) \| P_{k}(K)\right)\right) \Rightarrow Q_{1+k}^{-1}\left[Q_{1+k}(s)\right] \sigma \cap P_{1+k}(K)=\emptyset .
$$

(ii.b) The language $P_{2+k}(K) \subseteq\left(E_{2} \cup E_{k}\right)^{*}$ is observable with respect to $L\left(G_{2}\right)\left\|P_{k}(K)\right\| P_{k}^{1+k}\left(L\left(G_{1}\right) \| P_{k}(K)\right), E_{2+k, c}$, and $Q_{2+k}$; equivalently, for all $s \in P_{2+k}(K)$ and for all $\sigma \in E_{2+k, c}$,

$$
\left(s \sigma \notin P_{2+k}(K)\right) \text { and }\left(s \sigma \in L\left(G_{2}\right)\left\|P_{k}(K)\right\| P_{k}^{1+k}\left(L\left(G_{1}\right) \| P_{k}(K)\right)\right) \Rightarrow Q_{2+k}^{-1}\left[Q_{2+k}(s)\right] \sigma \cap P_{2+k}(K)=\emptyset .
$$

A procedure to determine whether a language is conditionally observable is provided in Section 6 . The following results are useful.

Lemma 4 ([4]). Let $P_{k}: E^{*} \rightarrow E_{k}^{*}$ be a natural projection, and let $L_{1} \subseteq E_{1}^{*}$ and $L_{2} \subseteq E_{2}^{*}$ be local languages over event sets $E_{1} \subseteq E$ and $E_{2} \subseteq E$, respectively, such that $E_{1} \cap E_{2} \subseteq E_{k}$. Then, $P_{k}\left(L_{1} \| L_{2}\right)=P_{k}\left(L_{1}\right) \| P_{k}\left(L_{2}\right)$.

Lemma 5 ([8, 9]). Let $L \subseteq E^{*}$ and $P_{k}: E^{*} \rightarrow E_{k}^{*}$ be a natural projection with $E_{k} \subseteq E$. Then, $L \| P_{k}(L)=L$.

Lemma 6 ([10]). Let $L_{i} \subseteq E_{i}^{*}(i=1,2)$, and let $E_{0}=E_{1} \cap E_{2}$. Define the natural projections $P_{i}:\left(E_{1} \cup E_{2}\right)^{*} \rightarrow E_{i}^{*}$ $(i=0,1,2)$ and $Q_{j}: E_{j}^{*} \rightarrow E_{0}^{*}(j=1,2)$. Then, for $i, j=1,2$ and $i \neq j, P_{i}\left(L_{1} \| L_{2}\right)=L_{i} \cap Q_{i}^{-1} Q_{j}\left(L_{j}\right)$.

Theorem 7. Consider the setting of Problem 1. There exists a set of supervisors $\left(S_{1}, S_{2}, S_{k}\right)$ such that

$$
L\left(S_{1} /\left[G_{1} \|\left(S_{k} / G_{k}\right)\right]\right)\left\|L\left(S_{2} /\left[G_{2} \|\left(S_{k} / G_{k}\right)\right]\right)\right\| L\left(S_{k} / G_{k}\right)=K
$$

if and only if (1) the specification language $K$ is conditionally controllable with respect to the set $\left(G_{1}, G_{2}, G_{k}\right)$ of generators and $\left(E_{1+k, u}, E_{2+k, u}, E_{k, u}\right)$ of locally uncontrollable events, and (2) conditionally observable with respect to the set $\left(G_{1}, G_{2}, G_{k}\right)$ of generators, $\left(E_{1+k, c}, E_{2+k, c}, E_{k, c}\right)$ of locally controllable events, and $\left(Q_{1+k}, Q_{2+k}, Q_{k}\right)$ of natural projections from $E_{i}^{*}$ to $E_{i, o}^{*}$, for $i=1+k, 2+k, k$.

Proof. To prove sufficiency, let $K$ be conditionally controllable with respect to $\left(G_{1}, G_{2}, G_{k}\right)$ and $\left(E_{1+k, u}, E_{2+k, u}, E_{k, u}\right)$, and conditionally observable with respect to $\left(G_{1}, G_{2}, G_{k}\right),\left(E_{1+k, c}, E_{2+k, c}, E_{k, c}\right)$, and $\left(Q_{1+k}, Q_{2+k}, Q_{k}\right)$. Equation (1) must be checked.

Since $K \subseteq L\left(G_{1}\left\|G_{2}\right\| G_{k}\right) \Rightarrow P_{k}(K) \subseteq P_{k}\left(L\left(G_{1}\right)\left\|L\left(G_{2}\right)\right\| L\left(G_{k}\right)\right) \subseteq L\left(G_{k}\right)$, and $P_{k}(K)$ is controllable with respect to $L\left(G_{k}\right)$ and $E_{k, u}$, and observable with respect to $L\left(G_{k}\right), E_{k, c}$, and $Q_{k}$, it follows from [11] that there exists a supervisor $S_{k}$ over the event set $E_{k}$ such that $L\left(S_{k} / G_{k}\right)=P_{k}(K)$.

Furthermore, $K \subseteq L\left(G_{1}\left\|G_{2}\right\| G_{k}\right)$ implies that

$$
\begin{aligned}
P_{1+k}(K) & \subseteq P_{1+k}\left(L\left(G_{1}\left\|G_{2}\right\| G_{k}\right)\right)=P_{1+k}\left(L\left(G_{1}\right) \| L\left(G_{k}\right)\right) \| P_{k \cap 2}\left(L\left(G_{2}\right)\right), \quad \text { by Lemma } 4, \\
& =L\left(G_{1}\right)\left\|P_{k \cap 2}\left(L\left(G_{2}\right)\right)\right\| L\left(G_{k}\right) .
\end{aligned}
$$

Then, $P_{1+k}(K) \subseteq L\left(G_{1}\right)\left\|P_{k \cap 2}\left(L\left(G_{2}\right)\right)\right\| L\left(G_{k}\right)$ and $P_{1+k}(K) \subseteq\left(P_{k}^{1+k}\right)^{-1} P_{k}(K)$ imply that

$$
\begin{aligned}
P_{1+k}(K) & \subseteq L\left(G_{1}\right)\left\|P_{k \cap 2}\left(L\left(G_{2}\right)\right)\right\| L\left(G_{k}\right) \| P_{k}(K) \\
& =L\left(G_{1}\right)\left\|P_{k \cap 2}\left(L\left(G_{2}\right)\right)\right\| L\left(G_{k}\right) \| L\left(S_{k} / G_{k}\right) \\
& =L\left(G_{1}\right)\left\|P_{k \cap 2}\left(L\left(G_{2}\right)\right)\right\| L\left(S_{k} / G_{k}\right), \quad \\
& =L\left(G_{1}\right)\left\|P_{k \cap 2}\left(L\left(G_{2}\right)\right)\right\| L\left(S_{k} / G_{k}\right) \| P_{k}(K) \quad \text { by } L\left(G_{k}\right) \| L\left(S_{k} / G_{k}\right)=L\left(S_{k} / G_{k}\right), \\
& =L\left(G_{1}\right)\left\|L\left(S_{k} / G_{k}\right)\right\| P_{k}\left(L\left(G_{2} \|\left(S_{k} / G_{k}\right)\right)\right), \quad \text { by } P_{k \cap 2}\left(L\left(G_{2}\right)\right) \| P_{k}(K)=P_{k}\left(L\left(G_{2} \|\left(S_{k} / G_{k}\right)\right)\right) .
\end{aligned}
$$

This, the assumption that the specification is conditionally controllable and conditionally observable, and [11] imply that there exists a supervisor $S_{1}$ such that $L\left(S_{1} /\left[G_{1}\left\|\left(S_{k} / G_{k}\right)\right\| P_{k}\left(G_{2} \|\left(S_{k} / G_{k}\right)\right)\right]\right)=P_{1+k}(K)$, where for a generator $G$ 
and a natural projection $P, P(G)$ denotes the minimal generator that generates the language $P(L(G))$, i.e., $L(P(G))=$ $P\left(L(G)\right.$ ) (see [1,4] for details). For (ii.b) of Definition 8, a similar argument shows that there exists a supervisor $S_{2}$ such that $L\left(S_{2} /\left[G_{2}\left\|\left(S_{k} / G_{k}\right)\right\| P_{k}\left(G_{1} \|\left(S_{k} / G_{k}\right)\right)\right]\right)=P_{2+k}(K)$.

In addition,

$$
\begin{aligned}
L\left(S_{i} /\left[G_{i}\left\|\left(S_{k} / G_{k}\right)\right\| P_{k}\left(G_{i} \|\left(S_{k} / G_{k}\right)\right)\right]\right) & =L\left(S_{i}\right)\left\|L\left(G_{i} \|\left(S_{k} / G_{k}\right)\right)\right\| P_{k}\left(L\left(G_{i} \|\left(S_{k} / G_{k}\right)\right)\right) \\
& =L\left(S_{i}\right) \| L\left(G_{i} \|\left(S_{k} / G_{k}\right)\right), \\
& =L\left(S_{i} /\left[G_{i} \|\left(S_{k} / G_{k}\right)\right]\right),
\end{aligned}
$$

by Lemma 5 ,

which follows from the properties of synchronous product. It is now sufficient to notice that

$$
\begin{aligned}
& L\left(S_{1} /\left[G_{1}\left\|\left(S_{k} / G_{k}\right)\right\| P_{k}\left(G_{2} \|\left(S_{k} / G_{k}\right)\right)\right]\right) \| L\left(S_{2} /\left[G_{2}\left\|\left(S_{k} / G_{k}\right)\right\| P_{k}\left(G_{1} \|\left(S_{k} / G_{k}\right)\right)\right]\right) \\
& =L\left(S_{1}\right)\left\|L\left(G_{1} \|\left(S_{k} / G_{k}\right)\right)\right\| P_{k}\left(L\left(G_{2} \|\left(S_{k} / G_{k}\right)\right)\right)\left\|L\left(S_{2}\right)\right\| L\left(G_{2} \|\left(S_{k} / G_{k}\right)\right) \| P_{k}\left(L\left(G_{1} \|\left(S_{k} / G_{k}\right)\right)\right) \\
& =L\left(S_{1}\right)\left\|L\left(G_{1} \|\left(S_{k} / G_{k}\right)\right)\right\| P_{k}\left(L\left(G_{1} \|\left(S_{k} / G_{k}\right)\right)\right)\left\|L\left(S_{2}\right)\right\| L\left(G_{2} \|\left(S_{k} / G_{k}\right)\right) \| P_{k}\left(L\left(G_{2} \|\left(S_{k} / G_{k}\right)\right)\right) \\
& =L\left(S_{1}\right)\left\|L\left(G_{1} \|\left(S_{k} / G_{k}\right)\right)\right\| L\left(S_{2}\right) \| L\left(G_{2} \|\left(S_{k} / G_{k}\right)\right), \quad \text { using }(*), \\
& =L\left(S_{1} /\left[G_{1} \|\left(S_{k} / G_{k}\right)\right]\right) \| L\left(S_{2} /\left[G_{2} \|\left(S_{k} / G_{k}\right)\right]\right),
\end{aligned}
$$

where the second equality is by the commutativity of the synchronous product. Summarized, we have shown that

$$
\begin{gathered}
L\left(S_{1} /\left[G_{1}\left\|\left(S_{k} / G_{k}\right)\right\| P_{k}\left(G_{2} \|\left(S_{k} / G_{k}\right)\right)\right]\right)\left\|L\left(S_{2} /\left[G_{2}\left\|\left(S_{k} / G_{k}\right)\right\| P_{k}\left(G_{1} \|\left(S_{k} / G_{k}\right)\right)\right]\right)\right\| L\left(S_{k} / G_{k}\right) \\
=L\left(S_{1} /\left[G_{1} \|\left(S_{k} / G_{k}\right)\right]\right)\left\|L\left(S_{2} /\left[G_{2} \|\left(S_{k} / G_{k}\right)\right]\right)\right\| L\left(S_{k} / G_{k}\right) .
\end{gathered}
$$

Finally, since $K$ is conditionally decomposable and the following equalities are proven above,

$$
\begin{aligned}
P_{1+k}(K) & =L\left(S_{1} /\left[G_{1}\left\|\left(S_{k} / G_{k}\right)\right\| P_{k}\left(G_{2} \|\left(S_{k} / G_{k}\right)\right)\right]\right) \\
P_{2+k}(K) & =L\left(S_{2} /\left[G_{2}\left\|\left(S_{k} / G_{k}\right)\right\| P_{k}\left(G_{1} \|\left(S_{k} / G_{k}\right)\right)\right]\right) \\
P_{k}(K) & =L\left(S_{k} / G_{k}\right),
\end{aligned}
$$

it follows that $L\left(S_{1} /\left[G_{1} \|\left(S_{k} / G_{k}\right)\right]\right)\left\|L\left(S_{2} /\left[G_{2} \|\left(S_{k} / G_{k}\right)\right]\right)\right\| L\left(S_{k} / G_{k}\right)=P_{1+k}(K)\left\|P_{2+k}(K)\right\| P_{k}(K)=K$. Thus, sufficiency is proven.

To prove necessity, projections $P_{k}, P_{1+k}$, and $P_{2+k}$ will be applied to Equation (1). Since all the supervisors cannot disable uncontrollable events, the closed-loop languages can be written as corresponding synchronous products. Thus, (1) can be rewritten as follows.

$$
\begin{aligned}
K & =L\left(S_{1}\right)\left\|L\left(G_{1}\right)\right\| L\left(S_{k}\right)\left\|L\left(G_{k}\right)\right\| L\left(S_{2}\right)\left\|L\left(G_{2}\right)\right\| L\left(S_{k}\right)\left\|L\left(G_{k}\right)\right\| L\left(S_{k}\right) \| L\left(G_{k}\right) \\
& =L\left(S_{1}\right)\left\|L\left(G_{1}\right)\right\| L\left(S_{2}\right)\left\|L\left(G_{2}\right)\right\| L\left(S_{k}\right) \| L\left(G_{k}\right),
\end{aligned}
$$

which yields after projecting by $P_{k}$ that

$$
\begin{aligned}
P_{k}(K) & =P_{k}\left(L\left(S_{1}\right)\left\|L\left(G_{1}\right)\right\| L\left(S_{2}\right)\left\|L\left(G_{2}\right)\right\| L\left(S_{k}\right) \| L\left(G_{k}\right)\right)=L\left(S_{k}\right) \| L\left(G_{k}\right) \cap P_{k}\left(L\left(S_{1}\right)\left\|L\left(G_{1}\right)\right\| L\left(S_{2}\right) \| L\left(G_{2}\right)\right) \\
& \subseteq L\left(S_{k}\right) \| L\left(G_{k}\right)=L\left(S_{k} / G_{k}\right) .
\end{aligned}
$$

On the other hand, we always have $L\left(S_{k} / G_{k}\right) \subseteq P_{k}(K)$ because $S_{k}$ is a supervisor that enforces the coordinator part of the specification $P_{k}(K)$. Hence, we have that $L\left(S_{k} / G_{k}\right)=P_{k}(K)$, which means, according to the basic theorem of supervisory control, that $P_{k}(K) \subseteq E_{k}^{*}$ is controllable with respect to $L\left(G_{k}\right)$ and $E_{k, u}$ and observable with respect to $L\left(G_{k}\right), E_{k, c}$, and $Q_{k}$, i.e., (i) of definitions of conditional controllability and conditional observability are satisfied.

Now, (ii.a) of conditional controllability is shown; (ii.b) is a symmetric condition. An application of the projection $P_{1+k}$ to (1) yields $P_{1+k}\left(L\left(S_{k} / G_{k}\right)\left\|L\left(S_{1} /\left[G_{1} \|\left(S_{k} / G_{k}\right)\right]\right)\right\| L\left(S_{2} /\left[G_{2} \|\left(S_{k} / G_{k}\right)\right]\right)\right)=P_{1+k}(K)$. Since $E_{1+k} \cap E_{2+k}=E_{k}$, and using the fact that $L\left(S_{2}\right) \| L\left(G_{2} \|\left(S_{k} / G_{k}\right)\right)=L\left(S_{2}\right) \cap L\left(G_{2} \|\left(S_{k} / G_{k}\right)\right)$ because both components are over the same event 
set $E_{2+k}$, we obtain that

$$
\begin{aligned}
P_{1+k}(K) & =L\left(S_{k} / G_{k}\right)\left\|L\left(S_{1} /\left[G_{1} \|\left(S_{k} / G_{k}\right)\right]\right)\right\| P_{1+k}\left(L\left(S_{2} /\left[G_{2} \|\left(S_{k} / G_{k}\right)\right]\right)\right) \\
& =L\left(S_{k} / G_{k}\right)\left\|L\left(S_{1} /\left[G_{1} \|\left(S_{k} / G_{k}\right)\right]\right)\right\| P_{1+k}\left(L\left(S_{2}\right) \| L\left(G_{2} \|\left(S_{k} / G_{k}\right)\right)\right) \\
& \subseteq L\left(S_{k} / G_{k}\right)\left\|L\left(S_{1} /\left[G_{1} \|\left(S_{k} / G_{k}\right)\right]\right)\right\| P_{1+k}\left(L\left(G_{2} \|\left(S_{k} / G_{k}\right)\right)\right) \\
& \subseteq L\left(S_{1} /\left[G_{1} \|\left(S_{k} / G_{k}\right)\right]\right) \| P_{1+k}\left(L\left(G_{2} \|\left(S_{k} / G_{k}\right)\right)\right) \\
& \subseteq L\left(S_{1} /\left[G_{1} \|\left(S_{k} / G_{k}\right)\right]\right) \\
& \subseteq P_{1+k}(K) .
\end{aligned}
$$

Using again the fact that the closed-loop behavior under admissible supervisors can be recast as a synchronous composition of the plant and the supervisor, we get $L\left(S_{1}\right)\left\|L\left(G_{1}\right)\right\| L\left(S_{k} / G_{k}\right) \| P_{1+k}\left(L\left(G_{2} \|\left(S_{k} / G_{k}\right)\right)\right)=P_{1+k}(K)$. The whole term after $L\left(S_{1}\right)$ can now be taken as a new plant $G_{1}\left\|\left(S_{k} / G_{k}\right)\right\| P_{1+k}\left(G_{2} \|\left(S_{k} / G_{k}\right)\right)$. According to the basic theorem of supervisory control it implies that $P_{1+k}(K)$ is controllable with respect to $G_{1}\left\|\left(S_{k} / G_{k}\right)\right\| P_{1+k}\left(G_{2} \|\left(S_{k} / G_{k}\right)\right)$ and $E_{1+k, u}$ and observable with respect to $G_{1}\left\|\left(S_{k} / G_{k}\right)\right\| P_{1+k}\left(G_{2} \|\left(S_{k} / G_{k}\right)\right), E_{1+k, c}$, and $Q_{1+k}$, i.e., (ii.a) of definitions of conditional controllability and conditional observability are satisfied, which was to be shown.

\section{Control synthesis of conditionally controllable and conditionally normal languages}

As discussed above, it is well known that supremal observable sublanguages do not exist in general and it is not hard to see that this is also the case of conditionally observable sublanguages. Therefore, this section introduces an analogous notion to normality, so called conditional normality, and proves that conditional normality along with conditional controllability are sufficient conditions for the specification language to solve Problem 1.

Definition 8. Consider the setting of Problem 1. Call the specification language $K \subseteq E^{*}$ conditionally normal for generators $\left(G_{1}, G_{2}, G_{k}\right)$ and for the natural projections $\left(Q_{1+k}, Q_{2+k}, Q_{k}\right)$, where $Q_{i}: E_{i}^{*} \rightarrow E_{i, o}^{*}$, for $i=1+k, 2+k, k$, if

(i) The language $P_{k}(K) \subseteq E_{k}^{*}$ is normal with respect to $L\left(G_{k}\right)$ and $Q_{k}$; equivalently,

$$
Q_{k}^{-1} Q_{k}\left(P_{k}(K)\right) \cap L\left(G_{k}\right)=P_{k}(K) .
$$

(ii.a) The language $P_{1+k}(K) \subseteq\left(E_{1} \cup E_{k}\right)^{*}$ is normal with respect to $L\left(G_{1}\right)\left\|P_{k}(K)\right\| P_{k}^{2+k}\left(L\left(G_{2}\right) \| P_{k}(K)\right)$ and $Q_{1+k}$; equivalently,

$$
Q_{1+k}^{-1} Q_{1+k}\left(P_{1+k}(K)\right) \cap L\left(G_{1}\right)\left\|P_{k}(K)\right\| P_{k}^{2+k}\left(L\left(G_{2}\right) \| P_{k}(K)\right)=P_{1+k}(K) .
$$

(ii.b) The language $P_{2+k}(K) \subseteq\left(E_{2} \cup E_{k}\right)^{*}$ is normal with respect to $L\left(G_{2}\right)\left\|P_{k}(K)\right\| P_{k}^{1+k}\left(L\left(G_{1}\right) \| P_{k}(K)\right)$ and $Q_{2+k}$; equivalently,

$$
Q_{2+k}^{-1} Q_{2+k}\left(P_{2+k}(K)\right) \cap L\left(G_{2}\right)\left\|P_{k}(K)\right\| P_{k}^{1+k}\left(L\left(G_{1}\right) \| P_{k}(K)\right)=P_{2+k}(K) .
$$

A procedure to determine whether a language is conditionally normal is provided in Section 6. To demonstrate that the definition is correct, it remains to show that $P_{i+k}(K) \subseteq L\left(G_{i}\right)\left\|P_{k}(K)\right\| P_{k}^{j+k}\left(L\left(G_{j}\right) \| P_{k}(K)\right)$, for $\{i, j\}=\{1,2\}, i \neq j$. This is shown in the following lemma.

Lemma 9. For $\{i, j\}=\{1,2\}, i \neq j$, it holds that $P_{i+k}(K) \subseteq L\left(G_{i}\right)\left\|P_{k}(K)\right\| P_{k}^{j+k}\left(L\left(G_{j}\right) \| P_{k}(K)\right)$.

Proof. By the definition, we need to show that $P_{i+k}(K) \subseteq\left(P_{i}^{i+k}\right)^{-1} L\left(G_{i}\right) \cap\left(P_{k}^{i+k}\right)^{-1} P_{k}(K) \cap\left(P_{k}^{i+k}\right)^{-1} P_{k}^{j+k}\left(L\left(G_{j}\right) \| P_{k}(K)\right)$. However, $P_{i+k}(K) \subseteq\left(P_{i}^{i+k}\right)^{-1} P_{i}^{i+k} P_{i+k}(K)=\left(P_{i}^{i+k}\right)^{-1} P_{i}(K)$ and $P_{i+k}(K) \subseteq\left(P_{k}^{i+k}\right)^{-1} P_{k}^{i+k} P_{i+k}(K)=\left(P_{k}^{i+k}\right)^{-1} P_{k}(K)$ imply that $P_{i+k}(K) \subseteq P_{i}(K) \| P_{k}(K)$, for $i=1,2$. In addition, the equality $P_{k}^{i+k} P_{i+k}(K)=P_{k}^{j+k} P_{j+k}(K)$ implies that $P_{i+k}(K) \subseteq$ $\left(P_{k}^{i+k}\right)^{-1} P_{k}^{i+k} P_{i+k}(K)=\left(P_{k}^{i+k}\right)^{-1} P_{k}^{j+k} P_{j+k}(K) \subseteq\left(P_{k}^{i+k}\right)^{-1} P_{k}^{j+k}\left(P_{j}(K) \| P_{k}(K)\right)$. Thus, we have shown that $P_{i+k}(K) \subseteq$ $P_{i}(K)\left\|P_{k}(K)\right\| P_{k}^{j+k}\left(P_{j}(K) \| P_{k}(K)\right)$. Finally, as $K \subseteq L\left(G_{1}\left\|G_{2}\right\| G_{k}\right)$, we obtain that $P_{i}(K) \subseteq P_{i}\left(L\left(G_{1}\left\|G_{2}\right\| G_{k}\right)\right) \subseteq L\left(G_{i}\right)$. This implies that $P_{i+k}(K) \subseteq P_{i}(K)\left\|P_{k}(K)\right\| P_{k}^{j+k}\left(P_{j}(K) \| P_{k}(K)\right) \subseteq L\left(G_{i}\right)\left\|P_{k}(K)\right\| P_{k}^{j+k}\left(L\left(G_{j}\right) \| P_{k}(K)\right)$, which was to be shown. 
Theorem 10. Consider the setting of Problem 1. If the specification language $K$ is conditionally controllable with respect to $\left(G_{1}, G_{2}, G_{k}\right)$ and $\left(E_{1+k, u}, E_{2+k, u}, E_{k, u}\right)$ of locally uncontrollable events, and conditionally normal with respect to $\left(G_{1}, G_{2}, G_{k}\right)$ and $\left(Q_{1+k}, Q_{2+k}, Q_{k}\right)$ of natural projections from $E_{i}^{*}$ to $E_{i, o}^{*}$, for $i=1+k, 2+k, k$, then there exist supervisors $S_{1}, S_{2}, S_{k}$ such that

$$
L\left(S_{1} /\left[G_{1} \|\left(S_{k} / G_{k}\right)\right]\right)\left\|L\left(S_{2} /\left[G_{2} \|\left(S_{k} / G_{k}\right)\right]\right)\right\| L\left(S_{k} / G_{k}\right)=K
$$

Proof. As normality implies observability, the proof of this theorem follows immediately from Theorem 7.

\section{Computation of supremal conditionally controllable and conditionally normal sublanguages}

So far, we have discussed conditions placed on the specification language under which a solution to Problem 1 exists. However, if the specification language does not satisfy these conditions, a supremal sublanguage that satisfies them is to be considered. In what follows, we present a procedure for computation of the supremal conditionally controllable and conditionally normal sublanguage for a given prefix-closed specification.

Theorem 11. The supremal conditionally controllable sublanguage of a given language $K$ always exists and is equal to the union of all conditionally controllable sublanguages of $\mathrm{K}$.

Proof. We show that conditional controllability is preserved by union. Let $I$ be an index set, and let $K_{i}, i \in I$, be conditionally controllable sublanguages of $K \subseteq L\left(G_{1}\left\|G_{2}\right\| G_{k}\right)$ with respect to generators $\left(G_{1}, G_{2}, G_{k}\right)$ and uncontrollable event sets $\left(E_{1+k, u}, E_{2+k, u}, E_{k, u}\right)$. We prove that $\bigcup_{i \in I} K_{i}$ is conditionally controllable with respect to those generators and uncontrollable event sets, i.e., the three items of the definition hold.

i) First, we prove that $P_{k}\left(\bigcup_{i \in I} K_{i}\right)$ is controllable with respect to $L\left(G_{k}\right)$ and $E_{k, u}$. To do this, note that

$$
P_{k}\left(\bigcup_{i \in I} K_{i}\right) E_{k, u} \cap L\left(G_{k}\right)=\bigcup_{i \in I}\left(P_{k}\left(K_{i}\right) E_{k, u} \cap L\left(G_{k}\right)\right) \subseteq \bigcup_{i \in I} P_{k}\left(K_{i}\right)=P_{k}\left(\bigcup_{i \in I} K_{i}\right),
$$

where the inclusion is by controllability of $P_{k}\left(K_{i}\right)$ with respect to $L\left(G_{k}\right)$ and $E_{k, u}, i \in I$.

ii) To prove the other statement, note first that

$$
L\left(G_{1}\right)\left\|P_{k}\left(\bigcup_{i \in I} K_{i}\right)\right\| P_{k}^{2+k}\left(L\left(G_{2}\right) \| P_{k}\left(\bigcup_{i \in I} K_{i}\right)\right)=L\left(G_{1}\right)\left\|P_{k}\left(\bigcup_{i \in I} K_{i}\right)\right\| P_{k}^{2+k}\left(L\left(G_{2}\right)\right)
$$

because $P_{k}^{2+k}\left(L\left(G_{2}\right) \| P_{k}\left(\cup_{i \in I} K_{i}\right)\right)=P_{k}^{2+k}\left(L\left(G_{2}\right)\right) \| P_{k}\left(\cup_{i \in I} K_{i}\right)$ by Lemma 4, and the second element is already included in the equation. Thus, we need to show that

$$
P_{1+k}\left(\bigcup_{i \in I} K_{i}\right) E_{1+k, u} \cap L\left(G_{1}\right)\left\|P_{k}\left(\bigcup_{i \in I} K_{i}\right)\right\| P_{k}^{2+k}\left(L\left(G_{2}\right)\right) \subseteq P_{1+k}\left(\bigcup_{i \in I} K_{i}\right) .
$$

However, it holds that

$$
\begin{aligned}
& P_{1+k}\left(\bigcup_{i \in I} K_{i}\right) E_{1+k, u} \cap L\left(G_{1}\right)\left\|P_{k}\left(\bigcup_{i \in I} K_{i}\right)\right\| P_{k}^{2+k}\left(L\left(G_{2}\right)\right) \\
&=\bigcup_{i \in I}\left(P_{1+k}\left(K_{i}\right) E_{1+k, u}\right) \cap \bigcup_{i \in I}\left(L\left(G_{1}\right)\left\|P_{k}\left(K_{i}\right)\right\| P_{k}^{2+k}\left(L\left(G_{2}\right)\right)\right) \\
&=\bigcup_{i \in I} \bigcup_{j \in I}\left(P_{1+k}\left(K_{i}\right) E_{1+k, u} \cap L\left(G_{1}\right)\left\|P_{k}\left(K_{j}\right)\right\| P_{k}^{2+k}\left(L\left(G_{2}\right)\right)\right) .
\end{aligned}
$$

For the sake of contradiction, assume that there are two different indexes $i, j \in I$ such that

$$
P_{1+k}\left(K_{i}\right) E_{1+k, u} \cap L\left(G_{1}\right)\left\|P_{k}\left(K_{j}\right)\right\| P_{k}^{2+k}\left(L\left(G_{2}\right)\right) \nsubseteq P_{1+k}\left(\bigcup_{i \in I} K_{i}\right) .
$$

Then, there exist $x \in P_{1+k}\left(K_{i}\right)$ and $u \in E_{1+k, u}$ such that $x u \in L\left(G_{1}\right)\left\|P_{k}\left(K_{j}\right)\right\| P_{k}^{2+k}\left(L\left(G_{2}\right)\right)$, and $x u \notin P_{1+k}\left(\cup_{i \in I} K_{i}\right)$. It follows that 
- $P_{k}(x) \in P_{k} P_{1+k}\left(K_{i}\right)=P_{k}\left(K_{i}\right)$,

- $P_{k}(x u) \in P_{k}\left(K_{j}\right)$, and

- $P_{k}(x u) \notin P_{k}\left(K_{i}\right)$; otherwise, if $P_{k}(x u) \in P_{k}\left(K_{i}\right)$, then $x u \in L\left(G_{1}\right)\left\|P_{k}\left(K_{i}\right)\right\| P_{k}\left(L\left(G_{2}\right)\right)$, and controllability of $P_{1+k}\left(K_{i}\right)$ with respect to $L\left(G_{1}\right)\left\|P_{k}\left(K_{i}\right)\right\| P_{k}\left(L\left(G_{2}\right)\right)$ implies that $x u \in P_{1+k}\left(K_{i}\right) \subseteq P_{1+k}\left(\cup_{i \in I} K_{i}\right)$, which is not true.

Assume that $u \notin E_{k, u}$. Then, $P_{k}(x u)=P_{k}(x) \in P_{k}\left(K_{i}\right)$, which does not hold. Thus, $u \in E_{k, u}$. As $P_{k}\left(K_{i}\right) \cup P_{k}\left(K_{j}\right) \subseteq$ $L\left(G_{k}\right)$, we get that

$$
P_{k}(x u)=P_{k}(x) u \in L\left(G_{k}\right) .
$$

However, controllability of $P_{k}\left(K_{i}\right)$ with respect to $L\left(G_{k}\right)$ and $E_{k, u}$ implies that $P_{k}(x) u=P_{k}(x u)$ is in $P_{k}\left(K_{i}\right)$. This is a contradiction.

iii) As the last item of the definition is proven in the same way, the theorem holds.

Theorem 12. The supremal conditionally normal sublanguage of a given language $K$ always exists and is equal to the union of all conditionally normal sublanguages of $K$.

Proof. We show that conditional normality is preserved by union. Let $I$ be an index set, and let $K_{i}, i \in I$, be conditionally normal sublanguages of $K \subseteq L\left(G_{1}\left\|G_{2}\right\| G_{k}\right)$ with respect to generators $\left(G_{1}, G_{2}, G_{k}\right)$ and the natural projections $\left(Q_{1+k}, Q_{2+k}, Q_{k}\right)$ to local observable event sets, see Fig. 1 . We prove that $\bigcup_{i \in I} K_{i}$ is conditionally normal with respect to those generators and natural projections, i.e., the three items of the definition hold.

i) First, note that $P_{k}\left(\bigcup_{i \in I} K_{i}\right)$ is normal with respect to $L\left(G_{k}\right)$ and $Q_{k}$ because

$$
Q_{k}^{-1} Q_{k} P_{k}\left(\bigcup_{i \in I} K_{i}\right) \cap L\left(G_{k}\right)=\bigcup_{i \in I}\left(Q_{k}^{-1} Q_{k} P_{k}\left(K_{i}\right) \cap L\left(G_{k}\right)\right)=\bigcup_{i \in I} P_{k}\left(K_{i}\right)=P_{k}\left(\bigcup_{i \in I} K_{i}\right),
$$

where the second equality is by normality of $P_{k}\left(K_{i}\right)$ with respect to $L\left(G_{k}\right)$ and $Q_{k}, i \in I$.

ii) To prove the other statement, by (1) we need to show that

$$
Q_{1+k}^{-1} Q_{1+k} P_{1+k}\left(\bigcup_{i \in I} K_{i}\right) \cap L\left(G_{1}\right)\left\|P_{k}\left(\bigcup_{i \in I} K_{i}\right)\right\| P_{k}^{2+k}\left(L\left(G_{2}\right)\right)=P_{1+k}\left(\bigcup_{i \in I} K_{i}\right) .
$$

However, it is true that $P_{1+k}\left(\cup_{i \in I} K_{i}\right) \subseteq Q_{1+k}^{-1} Q_{1+k} P_{1+k}\left(\cup_{i \in I} K_{i}\right) \cap L\left(G_{1}\right)\left\|P_{k}\left(\cup_{i \in I} K_{i}\right)\right\| P_{k}^{2+k}\left(L\left(G_{2}\right)\right)$, and that

$$
\begin{aligned}
Q_{1+k}^{-1} Q_{1+k} P_{1+k}\left(\bigcup_{i \in I} K_{i}\right) \cap & L\left(G_{1}\right)\left\|P_{k}\left(\bigcup_{i \in I} K_{i}\right)\right\| P_{k}^{2+k}\left(L\left(G_{2}\right)\right) \\
=\bigcup_{i \in I}\left(Q_{1+k}^{-1} Q_{1+k} P_{1+k}\left(K_{i}\right)\right) & \cap \bigcup_{i \in I}\left(L\left(G_{1}\right)\left\|P_{k}\left(K_{i}\right)\right\| P_{k}^{2+k}\left(L\left(G_{2}\right)\right)\right) \\
& =\bigcup_{i \in I} \bigcup_{j \in I}\left(Q_{1+k}^{-1} Q_{1+k} P_{1+k}\left(K_{i}\right) \cap L\left(G_{1}\right)\left\|P_{k}\left(K_{j}\right)\right\| P_{k}^{2+k}\left(L\left(G_{2}\right)\right)\right) .
\end{aligned}
$$

For the sake of contradiction, assume that there are two different indexes $i, j \in I$ such that

$$
Q_{1+k}^{-1} Q_{1+k} P_{1+k}\left(K_{i}\right) \cap L\left(G_{1}\right)\left\|P_{k}\left(K_{j}\right)\right\| P_{k}^{2+k}\left(L\left(G_{2}\right)\right) \nsubseteq P_{1+k}\left(\bigcup_{i \in I} K_{i}\right) .
$$

Then, there is $x \in Q_{1+k}^{-1} Q_{1+k} P_{1+k}\left(K_{i}\right)$ such that $x \in L\left(G_{1}\right)\left\|P_{k}\left(K_{j}\right)\right\| P_{k}^{2+k}\left(L\left(G_{2}\right)\right)$, and $x \notin P_{1+k}\left(\cup_{i \in I} K_{i}\right)$. It follows that

$$
P_{k}^{1+k}(x) \in P_{k}\left(K_{j}\right) \text { and } P_{k}^{1+k}(x) \notin P_{k}\left(K_{i}\right) ;
$$

otherwise, $P_{k}^{1+k}(x) \in P_{k}\left(K_{i}\right)$ implies that $x \in L\left(G_{1}\right)\left\|P_{k}\left(K_{i}\right)\right\| P_{k}\left(L\left(G_{2}\right)\right)$, and normality of $P_{1+k}\left(K_{i}\right)$ then implies that $x \in P_{1+k}\left(K_{i}\right) \subseteq P_{1+k}\left(\cup_{i \in I} K_{i}\right)$, which does not hold. As $P_{k}\left(K_{i}\right) \cup P_{k}\left(K_{j}\right) \subseteq L\left(G_{k}\right)$, we get that

$$
P_{k}^{1+k}(x) \in L\left(G_{k}\right) \text {. }
$$




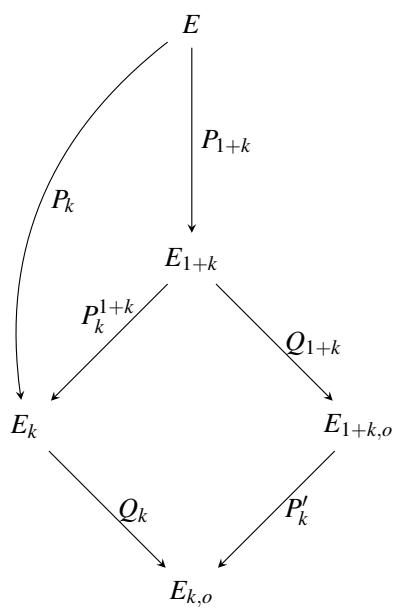

Figure 1: A commutative diagram of the natural projections.

However, as $x \in Q_{1+k}^{-1} Q_{1+k} P_{1+k}\left(K_{i}\right)$, there exists $w \in K_{i}$ such that $Q_{1+k}(x)=Q_{1+k} P_{1+k}(w)$. Thus, applying the natural projection $P_{k}^{\prime}: E_{1+k, o}^{*} \rightarrow E_{k, o}^{*}$, we get that $P_{k}^{\prime} Q_{1+k}(x)=P_{k}^{\prime} Q_{1+k} P_{1+k}(w)$. As it holds that $Q_{k} P_{k}^{1+k}=P_{k}^{\prime} Q_{1+k}$ and $Q_{k} P_{k}=P_{k}^{\prime} Q_{1+k} P_{1+k}$ (see Fig. 1), we have that

$$
Q_{k} P_{k}^{1+k}(x)=P_{k}^{\prime} Q_{1+k}(x)=P_{k}^{\prime} Q_{1+k} P_{1+k}(w)=Q_{k} P_{k}(w),
$$

i.e., $P_{k}^{1+k}(x) \in Q_{k}^{-1} Q_{k} P_{k}\left(K_{i}\right)$. By normality of $P_{k}\left(K_{i}\right)$ with respect to $L\left(G_{k}\right)$ and $Q_{k}$, we obtain that $P_{k}^{1+k}(x) \in P_{k}\left(K_{i}\right)$, which is a contradiction with (2).

iii) As the last item of the definition is proven in the same way, the theorem holds.

Given generators $G_{1}, G_{2}$, and $G_{k}$. For brevity we denote $L_{i}=L\left(G_{i}\right)$ in what follows, for $i=1,2, k$. In addition, let

$$
\sup \operatorname{cCN}\left(K, L,\left(E_{1+k, u}, E_{2+k, u}, E_{k, u}\right),\left(Q_{1+k}, Q_{2+k}, Q_{k}\right)\right)
$$

denote the supremal conditionally controllable and conditionally normal sublanguage of the specification language $K$ with respect to the plant language $L=L\left(G_{1}\left\|G_{2}\right\| G_{k}\right)$, the sets of uncontrollable events $\left(E_{1+k, u}, E_{2+k, u}, E_{k, u}\right)$, and the natural projections $\left(Q_{1+k}, Q_{2+k}, Q_{k}\right)$, where $Q_{i}: E_{i}^{*} \rightarrow E_{i, o}^{*}$, for $i=1+k, 2+k, k$. The following auxiliary lemmas will be useful.

Lemma 13. Let $L_{1} \subseteq E_{1}^{*}, L_{2} \subseteq E_{2}^{*}$, and $E_{1} \cap E_{2} \subseteq E_{k}$. Then, $P_{k}\left(L_{1} \| L_{2}\right)=P_{k}^{1+k}\left(P_{1}^{1+k}\right)^{-1}\left(L_{1}\right) \cap P_{k}^{2+k}\left(P_{2}^{2+k}\right)^{-1}\left(L_{2}\right)$.

Proof. This follows from Lemma 4, the definition of the synchronous product, and Proposition 4.2(6) in [10] showing the commutativity $\left(P_{i \cap k}^{k}\right)^{-1} P_{i \cap k}^{i}=P_{k}^{i+k}\left(P_{i}^{i+k}\right)^{-1}$, for $i=1,2$. Specifically, in turn we have

$$
P_{k}\left(L_{1} \| L_{2}\right)=P_{k}\left(L_{1}\right) \| P_{k}\left(L_{2}\right)=\left(P_{1 \cap k}^{k}\right)^{-1} P_{1 \cap k}^{1}\left(L_{1}\right) \cap\left(P_{2 \cap k}^{k}\right)^{-1} P_{2 \cap k}^{2}\left(L_{2}\right)=P_{k}^{1+k}\left(P_{1}^{1+k}\right)^{-1}\left(L_{1}\right) \cap P_{k}^{2+k}\left(P_{2}^{2+k}\right)^{-1}\left(L_{2}\right),
$$

which proves the lemma.

Lemma 14 ([8]). Let $E=E_{1} \cup E_{2}$ be event sets, and let $L_{1} \subseteq E_{1}^{*}$ and $L_{2} \subseteq E_{2}^{*}$ be two languages. Let $P_{i}: E^{*} \rightarrow E_{i}^{*}$ be natural projections, for $i=1,2$. Let $A \subseteq E^{*}$ be a language such that $P_{1}(A) \subseteq L_{1}$ and $P_{2}(A) \subseteq L_{2}$. Then, $A \subseteq L_{1} \| L_{2}$.

Lemma 15 ([8]). Let $K \subseteq L \subseteq M$ be languages over an event set $E$ such that $K$ is controllable with respect to $L$ and $E_{u}$, and $L$ is controllable with respect to $M$ and $E_{u}$. Then, $K$ is controllable with respect to $M$ and $E_{u}$.

Lemma 16. Let $K \subseteq L \subseteq M$ be prefix-closed languages such that $K$ is normal with respect to $L$ and $Q$, and $L$ is normal with respect to $M$ and $Q$. Then, $K$ is normal with respect to $M$ and $Q$. 
Proof. We know that $Q^{-1} Q(K) \cap L=K$ and $Q^{-1} Q(L) \cap M=L$. Then, $Q^{-1} Q(K) \cap M \subseteq Q^{-1} Q(L) \cap M=L$. This implies that $Q^{-1} Q(K) \cap M=\left(Q^{-1} Q(K) \cap M\right) \cap L=\left(Q^{-1} Q(K) \cap L\right) \cap M=K \cap M=K$.

Lemma 17. Let $Q: E^{*} \rightarrow E_{o}^{*}, P_{k}: E^{*} \rightarrow E_{k}^{*}$, and $Q_{k}: E_{k}^{*} \rightarrow E_{k, o}^{*}$ be natural projections. Then, for every language $M \subseteq E_{k}^{*}$, the inclusion $Q^{-1} Q P_{k}^{-1}(M) \subseteq P_{k}^{-1} Q_{k}^{-1} Q_{k}(M)$ holds.

Proof. Let $M \subseteq E_{k}^{*}$ be a language, then $Q P_{k}^{-1}(X) \subseteq Q P_{k}^{-1} Q_{k}^{-1} Q_{k}(X)=Q\left(Q_{k} P_{k}\right)^{-1} Q_{k}(X)=Q\left(P_{k}^{\prime} Q\right)^{-1} Q_{k}(X)=$ $Q Q^{-1}\left(P_{k}^{\prime}\right)^{-1} Q_{k}(X)=\left(P_{k}^{\prime}\right)^{-1} Q_{k}(X)$, where $P_{k}^{\prime}: E_{o}^{*} \rightarrow E_{k, o}^{*}$ is a restriction of the projection $P_{k}$ to $E_{o}^{*}$. This implies that $Q^{-1} Q P_{k}^{-1}(X) \subseteq Q^{-1}\left(P_{k}^{\prime}\right)^{-1} Q_{k}(X)=\left(P_{k}^{\prime} Q\right)^{-1} Q_{k}(X)=\left(Q_{k} P_{k}\right)^{-1} Q_{k}(X)=P_{k}^{-1} Q_{k}^{-1} Q_{k}(X)$, which was to be shown.

The following conditions are required in the main result of this section. The reader is referred to [10, 12] for more details.

Definition 18. The natural projection $P_{k}: E^{*} \rightarrow E_{k}^{*}$, where $E_{k} \subseteq E$, is an $L$-observer for $L \subseteq E^{*}$ if, for all $t \in P(L)$ and $s \in \bar{L}, P(s)$ is a prefix of $t$ implies that there exists $u \in E^{*}$ such that $s u \in L$ and $P(s u)=t$.

Definition 19. The natural projection $P_{k}: E^{*} \rightarrow E_{k}^{*}$, where $E_{k} \subseteq E$, is output control consistent (OCC) for $L \subseteq E^{*}$ if for every $s \in \bar{L}$ of the form

$$
s=\sigma_{1} \ldots \sigma_{\ell} \quad \text { or } \quad s=s^{\prime} \sigma_{0} \sigma_{1} \ldots \sigma_{\ell}, \quad \ell \geq 1,
$$

where $s^{\prime} \in E^{*}, \sigma_{0}, \sigma_{\ell} \in E_{k}$, and $\sigma_{i} \in E \backslash E_{k}$, for $i=1,2, \ldots, \ell-1$, if $\sigma_{\ell} \in E_{u}$, then $\sigma_{i} \in E_{u}$, for all $i=1,2, \ldots, \ell-1$.

Note that OCC can be replaced by a similar condition called local control consistency (LCC) discussed in [13, 14].

Theorem 20. Consider the setting of Problem 1. Define the local languages

$$
\begin{aligned}
\sup \mathrm{CN}_{k} & =\sup \mathrm{CN}\left(P_{k}(K), L\left(G_{k}\right), E_{k, u}, Q_{k}\right), \\
\sup \mathrm{CN}_{1+k} & =\sup \mathrm{CN}\left(P_{1+k}(K), L\left(G_{1}\right) \| \sup \mathrm{CN}_{k}, E_{1+k, u}, Q_{1+k}\right), \\
\sup \mathrm{CN}_{2+k} & =\sup \mathrm{CN}\left(P_{2+k}(K), L\left(G_{2}\right) \| \sup \mathrm{CN}_{k}, E_{2+k, u}, Q_{2+k}\right) .
\end{aligned}
$$

Let the projection $P_{k}^{i+k}$ be an $\left(P_{i}^{i+k}\right)^{-1} L\left(G_{i}\right)$-observer and $O C C$ for $\left(P_{i}^{i+k}\right)^{-1} L\left(G_{i}\right)$, for $i=1,2$. Assume that the language $P_{k}^{1+k}\left(\sup \mathrm{CN}_{1+k}\right) \cap P_{k}^{2+k}\left(\sup \mathrm{CN}_{2+k}\right)$ is normal with respect to $L\left(G_{k}\right)$ and $Q_{k}$. Then,

$$
\sup \mathrm{CN}_{k}\left\|\sup \mathrm{CN}_{1+k}\right\| \sup \mathrm{CN}_{2+k}=\operatorname{supcCN}\left(K, L,\left(E_{1+k, u}, E_{2+k, u}, E_{k, u}\right),\left(Q_{1+k}, Q_{2+k}, Q_{k}\right)\right) \text {. }
$$

Proof. To prove the theorem, we first denote the left-hand side and the right-hand side as

$$
M=\sup \mathrm{CN}_{k}\left\|\sup \mathrm{CN}_{1+k}\right\| \sup \mathrm{CN}_{2+k} \quad \text { and } \quad \operatorname{supcCN}=\operatorname{supcCN}\left(K, L,\left(E_{1+k, u}, E_{2+k, u}, E_{k, u}\right),\left(Q_{1+k}, Q_{2+k}, Q_{k}\right)\right),
$$

respectively, and we denote $L=L\left(G_{1}\left\|G_{2}\right\| G_{k}\right)$ and $L_{i}=L\left(G_{i}\right), i=1,2, k$. To show that the inclusion $M \subseteq$ sup cCN holds, we need to show that

1. $M \subseteq K$,

2. $M$ is conditionally controllable with respect to $L$ and $\left(E_{1+k, u}, E_{2+k, u}, E_{k, u}\right)$, and

3. $M$ is conditionally normal with respect to $L$ and $\left(Q_{1+k}, Q_{2+k}, Q_{k}\right)$.

1. Note that $M=\sup \mathrm{CN}_{k}\left\|\sup \mathrm{CN}_{1+k}\right\| \sup \mathrm{CN}_{2+k} \subseteq P_{k}(K)\left\|P_{1+k}(K)\right\| P_{2+k}(K)=K$ since $K$ is conditionally decomposable. Thus, $M \subseteq K$ holds true.

2. For a proof showing that $M$ is conditionally controllable with respect to $L$ and $\left(E_{1+k, u}, E_{2+k, u}, E_{k, u}\right)$, the reader is referred to [8].

3. Thus, it remains to prove that $M$ is conditionally normal with respect to $L$ and $\left(Q_{1+k}, Q_{2+k}, Q_{k}\right)$. To do this, we need to show the following three properties of Definition 8:

(I) $Q_{k}^{-1} Q_{k}\left(P_{k}(M)\right) \cap L\left(G_{k}\right)=P_{k}(M)$, 
(II) $Q_{1+k}^{-1} Q_{1+k}\left(P_{1+k}(M)\right) \cap L\left(G_{1}\right)\left\|P_{k}(M)\right\| P_{k}^{2+k}\left(L\left(G_{2}\right) \| P_{k}(M)\right)=P_{1+k}(M)$, and

(III) $Q_{2+k}^{-1} Q_{2+k}\left(P_{2+k}(M)\right) \cap L\left(G_{2}\right)\left\|P_{k}(M)\right\| P_{k}^{1+k}\left(L\left(G_{1}\right) \| P_{k}(M)\right)=P_{2+k}(M)$.

As the last two properties are similar, we prove only (II).

(I) To prove $Q_{k}^{-1} Q_{k}\left(P_{k}(M)\right) \cap L\left(G_{k}\right)=P_{k}(M)$, note that

$$
P_{k}(M)=\sup \mathrm{CN}_{k} \cap P_{k}^{1+k}\left(\sup \mathrm{CN}_{1+k}\right) \cap P_{k}^{2+k}\left(\sup \mathrm{CN}_{2+k}\right)=P_{k}^{1+k}\left(\sup \mathrm{CN}_{1+k}\right) \cap P_{k}^{2+k}\left(\sup \mathrm{CN}_{2+k}\right) \text {, }
$$

where the first equality follows from Lemma 4 by replacing the synchronous product with the intersection, and the other follows from the fact that $\sup \mathrm{CN}_{i+k} \subseteq L\left(G_{i}\right) \| \sup \mathrm{CN}_{k} \subseteq\left(P_{k}^{i+k}\right)^{-1}\left(\sup \mathrm{CN}_{k}\right)$. Thus, by the assumption, $P_{k}(M)$ is normal with respect to $L\left(G_{k}\right)$ and $Q_{k}$, i.e., (I) holds true.

(II) Now, we show the other property, namely

$$
Q_{1+k}^{-1} Q_{1+k}\left(P_{1+k}(M)\right) \cap L\left(G_{1}\right)\left\|P_{k}(M)\right\| P_{k}^{2+k}\left(L\left(G_{2}\right) \| P_{k}(M)\right)=P_{1+k}(M) .
$$

The inclusion $\supseteq$ is proven as in Lemma 9. Thus, it remains to show the other inclusion. First, note that by Lemma 6 and the definition of synchronous product we obtain that

$$
P_{1+k}(M)=\left(P_{k}^{1+k}\right)^{-1}\left(\sup \mathrm{CN}_{k}\right) \cap \sup \mathrm{CN}_{1+k} \cap\left(P_{k}^{1+k}\right)^{-1} P_{k}^{2+k}\left(\sup \mathrm{CN}_{2+k}\right) .
$$

Assume that $x \in Q_{1+k}^{-1} Q_{1+k}\left(P_{1+k}(M)\right) \cap L\left(G_{1}\right)\left\|P_{k}(M)\right\| P_{k}^{2+k}\left(L\left(G_{2}\right) \| P_{k}(M)\right)$. Then, $P_{1+k}(M) \subseteq \sup \mathrm{CN}_{1+k}$ and $P_{k}(M) \subseteq$ sup $\mathrm{CN}_{k}$ imply that

$$
\begin{aligned}
Q_{1+k}^{-1} Q_{1+k}\left(P_{1+k}(M)\right) \cap L\left(G_{1}\right)\left\|P_{k}(M)\right\| P_{k}^{2+k}\left(L\left(G_{2}\right) \|\right. & \left.P_{k}(M)\right) \\
& \subseteq Q_{1+k}^{-1} Q_{1+k}\left(\operatorname{sup~CN}_{1+k}\right) \cap L\left(G_{1}\right)\left\|P_{k}(M)\right\| P_{k}^{2+k}\left(L\left(G_{2}\right) \| P_{k}(M)\right) \\
& \subseteq Q_{1+k}^{-1} Q_{1+k}\left(\sup \mathrm{CN}_{1+k}\right) \cap L\left(G_{1}\right) \| \sup \mathrm{CN}_{k}=\operatorname{sup~CN}_{1+k} .
\end{aligned}
$$

Thus, we have shown that $x \in \sup \mathrm{CN}_{1+k}$. In addition, it is satisfied that $P_{k}^{1+k}(x) \in P_{k}^{2+k}\left(L\left(G_{2}\right) \| P_{k}(M)\right) \subseteq P_{k}(M) \subseteq$ $P_{k}^{2+k}\left(\sup \mathrm{CN}_{2+k}\right)$, which implies that $x \in\left(P_{k}^{1+k}\right)^{-1} P_{k}^{2+k}\left(\sup \mathrm{CN}_{2+k}\right)$. Furthermore, $P_{k}^{1+k}(x) \in P_{k}(M) \subseteq \sup \mathrm{CN}_{k}$ implies that $x \in\left(P_{k}^{1+k}\right)^{-1}\left(\sup \mathrm{CN}_{k}\right)$. Hence, $x \in P_{1+k}(M)$. As (III) is proven analogously, we have shown that $M \subseteq \sup \mathrm{cCN}$.

To prove the opposite inclusion, sup $\mathrm{cN} \subseteq M$, by Lemma 14 it is sufficient to show that

- $P_{k}(\operatorname{supcCN}) \subseteq \sup \mathrm{CN}_{k}$ and

- $P_{i+k}(\sup \mathrm{cCN}) \subseteq \sup \mathrm{CN}_{i+k}$, for $i=1,2$.

To prove this, note that $P_{k}(\sup \mathrm{cCN}) \subseteq P_{k}(K)$ is controllable with respect to $L\left(G_{k}\right)$ and $E_{k, u}$ and normal with respect to $L\left(G_{k}\right)$ and $Q_{k}$, which implies that $P_{k}(\operatorname{supcCN}) \subseteq \sup \mathrm{CN}_{k}$ is satisfied. Furthermore, $P_{1+k}(\operatorname{supcCN}) \subseteq P_{1+k}(K)$ is controllable with respect to $L_{1}\left\|P_{k}(\sup c \mathrm{CN})\right\| P_{k}^{2+k}\left(L_{2} \| P_{k}(\operatorname{supcCN})\right)$ and $E_{1+k, u}$ and normal with respect to the same language and $Q_{1+k}$. By Lemma 13, $P_{k}(\operatorname{supcCN}) \subseteq \sup \mathrm{CN}_{k} \subseteq P_{k}(L) \subseteq P_{k}^{2+k}\left(P_{2}^{2+k}\right)^{-1}\left(L_{2}\right)$. The following holds:

$$
\begin{aligned}
L_{1}\left\|P_{k}(\sup \mathrm{cCN})\right\| P_{k}^{2+k}\left(L_{2} \| P_{k}(\sup \mathrm{cCN})\right) & =L_{1}\left\|P_{k}(\operatorname{supcCN})\right\|\left[P_{k}(\operatorname{supcCN}) \cap P_{k}^{2+k}\left(P_{2}^{2+k}\right)^{-1}\left(L_{2}\right)\right] \\
& =L_{1}\left\|P_{k}(\sup \mathrm{cCN})\right\| P_{k}(\sup \mathrm{cCN}) \\
& =L_{1} \| P_{k}(\sup \mathrm{cCN}) .
\end{aligned}
$$

Since $P_{k}(\operatorname{supcCN})$ is controllable with respect to $L\left(G_{k}\right)$ and $E_{k, u}$, and normal with respect to $L\left(G_{k}\right)$ and $Q_{k}$, it is also controllable with respect to $\sup \mathrm{CN}_{k} \subseteq L\left(G_{k}\right)$ and $E_{k, u}$, and normal with respect to sup $\mathrm{CN}_{k}$ and $Q_{k}$ because $P_{k}(\operatorname{supcCN}) \subseteq \sup \mathrm{CN}_{k}$. As $P_{1+k}(\operatorname{supcCN})$ is controllable with respect to $L_{1} \| P_{k}(\operatorname{supcCN})$ and $E_{1+k, u}$, and $L_{1} \| P_{k}(\operatorname{supcCN})$ is controllable with respect to $L_{1} \| \sup \mathrm{CN}_{k}$ and $E_{1+k, u}$ by [10, Proposition 4.6] (since all the languages under consideration are prefix-closed), Lemma 15 implies that $P_{1+k}(\operatorname{supcCN})$ is controllable with respect 
to $L_{1} \| \sup \mathrm{CN}_{k}$ and $E_{1+k, u}$. Furthermore, as $P_{1+k}(\operatorname{supcCN})$ is normal with respect to $L_{1} \| P_{k}(\operatorname{supcCN})$ and $Q_{1+k}$, and $L_{1} \| P_{k}$ (sup cCN) is normal with respect to $L_{1} \| \sup \mathrm{CN}_{k}$ and $Q_{1+k}$ because (using Lemma 17)

$$
\begin{aligned}
& Q_{1+k}^{-1} Q_{1+k}\left(L_{1} \| P_{k}(\operatorname{supcCN})\right) \cap L_{1} \| \sup \mathrm{CN}_{k} \\
&=Q_{1+k}^{-1} Q_{1+k}\left(\left(P_{1}^{1+k}\right)^{-1}\left(L_{1}\right) \cap\left(P_{k}^{1+k}\right)^{-1} P_{k}(\operatorname{supcCN})\right) \cap\left(P_{1}^{1+k}\right)^{-1}\left(L_{1}\right) \cap\left(P_{k}^{1+k}\right)^{-1}\left(\sup \mathrm{CN}_{k}\right) \\
& \subseteq Q_{1+k}^{-1} Q_{1+k}\left(P_{1}^{1+k}\right)^{-1}\left(L_{1}\right) \cap Q_{1+k}^{-1} Q_{1+k}\left(P_{k}^{1+k}\right)^{-1} P_{k}(\operatorname{supcCN}) \cap\left(P_{1}^{1+k}\right)^{-1}\left(L_{1}\right) \cap\left(P_{k}^{1+k}\right)^{-1}\left(\sup _{\mathrm{CN}}\right) \\
&=\left(P_{1}^{1+k}\right)^{-1}\left(L_{1}\right) \cap Q_{1+k}^{-1} Q_{1+k}\left(P_{k}^{1+k}\right)^{-1} P_{k}(\operatorname{supcCN}) \cap\left(P_{k}^{1+k}\right)^{-1}\left(\sup \mathrm{CN}_{k}\right) \\
& \subseteq\left(P_{1}^{1+k}\right)^{-1}\left(L_{1}\right) \cap\left(P_{k}^{1+k}\right)^{-1} Q_{k}^{-1} Q_{k} P_{k}(\operatorname{supcCN}) \cap\left(P_{k}^{1+k}\right)^{-1}\left(\sup \mathrm{CN}_{k}\right) \\
&=\left(P_{1}^{1+k}\right)^{-1}\left(L_{1}\right) \cap\left(P_{k}^{1+k}\right)^{-1}\left(Q_{k}^{-1} Q_{k} P_{k}(\operatorname{supcCN}) \cap \sup \mathrm{CN}_{k}\right) \\
&=\left(P_{1}^{1+k}\right)^{-1}\left(L_{1}\right) \cap\left(P_{k}^{1+k}\right)^{-1} P_{k}(\sup \mathrm{cCN}) \\
&=L_{1} \| P_{k}(\sup \mathrm{cN}) .
\end{aligned}
$$

Then, Lemma 16 implies that $P_{1+k}(\operatorname{supcCN})$ is normal with respect to $L_{1} \|$ sup $\mathrm{CN}_{k}$ and $Q_{1+k}$. Thus, we have shown that $P_{1+k}(\sup c \mathrm{CN}) \subseteq \sup \mathrm{CN}_{1+k}$. The case of the property (ii.b) is proven analogously. Hence, sup cCN $\subseteq M$ and the proof is complete.

Remark 21. The assumption that the language $P_{k}^{1+k}\left(\sup \mathrm{CN}_{1+k}\right) \cap P_{k}^{2+k}\left(\sup \mathrm{CN}_{2+k}\right)$ is normal is rather technical. If $E_{k}=E_{k, o}=E_{k} \cap E_{o}$, then the projection $Q_{k}$ is identity and the condition is trivially satisfied. However, the example below (see Section 6.1) demonstrates that the condition can also be satisfied although $E_{k}$ and $E_{k, o}$ do not coincide. On the other hand, if $E_{o} \subseteq E_{k}, P_{k}^{1+k}\left(\operatorname{sup~} \mathrm{CN}_{1+k}\right) \cap P_{k}^{2+k}\left(\operatorname{sup~} \mathrm{CN}_{2+k}\right)$ is normal with respect to $P_{k}\left(L_{1} \| L_{2}\right) \| L_{k}$. Thus, for the coordinator defined as $L_{k}=P_{k}\left(L_{1} \| L_{2}\right)$ discussed in the example below, the technical assumption is satisfied. The proof is as follows: $E_{o} \subseteq E_{k}$ implies $Q_{k} P_{k}=Q$. Then, normality of $K$ with respect to $L$ and $Q$ implies normality of $P_{k}(K)$ with respect to $P_{k}(L)$ and $Q_{k}$. To see this, let $t \in P_{k}(L), t^{\prime} \in P_{k}(K)$, and $Q_{k}(t)=Q_{k}\left(t^{\prime}\right)$. There exist $s \in L$ and $s^{\prime} \in K$ such that $P_{k}(s)=t$ and $P_{k}\left(s^{\prime}\right)=t^{\prime}$. Thus, $Q(s)=Q_{k} P_{k}(s)=Q_{k}(t)=Q_{k}\left(t^{\prime}\right)=Q_{k} P_{k}\left(s^{\prime}\right)=Q\left(s^{\prime}\right)$. Now, normality of $K$ with respect to $L$ and $Q$ implies $s \in K$, i.e., $t=P_{k}(s) \in P_{k}(K)$. Note that $\operatorname{sup~CN}_{1+k}$ is normal with respect to $L_{1} \| \sup \mathrm{CN}_{k}$ and $Q_{1+k}$, sup $\mathrm{CN}_{k}$ is normal with respect to $L_{k}$ and $Q_{k}$, and $L_{1}$ is normal with respect to $L_{1}$ and $Q_{1}$. This implies that $L_{1} \| \sup \mathrm{CN}_{k}$ is normal with respect to $L_{1} \| L_{k}$ and $Q_{1+k}$. Lemma 16 then implies that sup $\mathrm{CN}_{1+k}$ is normal with respect to $L_{1} \| L_{k}$ and $Q_{1+k}$. The same arguments show that sup $\mathrm{CN}_{2+k}$ is normal with respect to $L_{2} \| L_{k}$ and $Q_{2+k}$. Now, two applications of the preservation of normality under projection yield normality of $P_{k}^{i+k}\left(\operatorname{sup~} \mathrm{CN}_{i+k}\right)$ with respect to $P_{k}\left(L_{i} \| L_{k}\right)=L_{k} \| P_{k}\left(L_{i}\right)$ and $Q_{k}$, for $i=1$, 2. Then, $P_{k}^{1+k}\left(\sup \mathrm{CN}_{1+k}\right) \cap P_{k}^{2+k}\left(\sup \mathrm{CN}_{2+k}\right)$ is normal with respect to $L_{k}\left\|P_{k}\left(L_{1}\right)\right\| P_{k}\left(L_{2}\right)=L_{k}$ and $Q_{k}$ as required in the technical condition.

Let us mention that even if $P_{k}\left(\sup \mathrm{CN}_{1+k}\right) \cap P_{k}\left(\sup \mathrm{CN}_{2+k}\right)$ fails to be normal with respect to $L_{k}$ and $Q_{k}$, and the equality in Theorem 20 does not need to hold, it does not mean that our approach is useless. In fact, our procedure to compute the supremal normal sublanguage as the composition of corresponding supremal normal sublanguages over the alphabets $E_{k}, E_{1} \cup E_{k}$, and $E_{2} \cup E_{k}$ is natural and we always compute a normal sublanguage of $L_{1}\left\|L_{2}\right\| L_{k}$ using the computation scheme. Indeed, it follows by the same argument as the proof of normality of $P_{k}\left(\sup \mathrm{CN}_{1+k}\right) \cap$ $P_{k}\left(\sup \mathrm{CN}_{2+k}\right)$ with respect to $L_{k}$ and $Q_{k}$ in the special case $E_{o} \subseteq E_{k}$ discussed above. Namely, sup $\mathrm{CN}_{k}$ is by definition normal with respect to $L_{k}$ and $Q_{k}$, and for $i=1,2$ we have that $\sup \mathrm{CN}_{i+k}$ is normal with respect to $L_{i} \| L_{k}$ and $Q_{i+k}$. Since we deal with prefix-closed (hence nonconflicting) languages, this gives that $M=\sup \mathrm{CN}_{k}\left\|\sup \mathrm{CN}_{1+k}\right\| \sup \mathrm{CN}_{2+k}$ is normal with respect to $L_{k}\left\|\left(L_{1} \| L_{k}\right)\right\|\left(L_{2} \| L_{k}\right)=L_{1}\left\|L_{2}\right\| L_{k}$. This is because normality (as well as controllability) of prefix-closed languages is preserved by the parallel composition under a very mild assumption that all shared events have the same observability status in all subsystems that share them. Formally, $E_{o, 2} \cap E_{1}=E_{2} \cap E_{o, 1}$, which is automatically satisfied because we defined locally observable events as $E_{o, i}=E_{o} \cap E_{i}$.

As the assumption that the projection $P_{k}^{i+k}$ is an $\left(P_{i}^{i+k}\right)^{-1} L\left(G_{i}\right)$-observer and OCC for $\left(P_{i}^{i+k}\right)^{-1} L\left(G_{i}\right)$, for $i=1,2$, is required only for controllability, we have the following corollary. Let $\sup \mathrm{N}(K, L, Q)$ denote the supremal normal sublanguage of $K$ with respect to $L$ and $Q$. 
Corollary 22. Consider the setting of Problem 1. Define the local languages

$$
\begin{aligned}
\sup \mathrm{N}_{k} & =\sup \mathrm{N}\left(P_{k}(K), L\left(G_{k}\right), Q_{k}\right), \\
\sup \mathrm{N}_{1+k} & =\sup \mathrm{N}\left(P_{1+k}(K), L\left(G_{1}\right) \| \sup \mathrm{N}_{k}, Q_{1+k}\right), \\
\sup \mathrm{N}_{2+k} & =\sup \mathrm{N}\left(P_{2+k}(K), L\left(G_{2}\right) \| \sup \mathrm{N}_{k}, Q_{2+k}\right) .
\end{aligned}
$$

Assume that the language $P_{k}^{1+k}\left(\sup \mathrm{N}_{1+k}\right) \cap P_{k}^{2+k}\left(\operatorname{sup~} \mathrm{N}_{2+k}\right)$ is normal with respect to $L\left(G_{k}\right)$ and $Q_{k}$. Then,

$$
\sup \mathrm{N}_{k}\left\|\sup \mathrm{N}_{1+k}\right\| \sup \mathrm{N}_{2+k}=\sup \mathrm{cN}\left(K, L,\left(Q_{1+k}, Q_{2+k}, Q_{k}\right)\right) \text {. }
$$

The minimal cardinality of the coordinator event set depends on the specification language. If we assume that the projections are observers, the state size of the computed models is guaranteed to be no larger than that of the original models. In a typical situation, the projected models are significantly smaller than the original models. The reader is referred to $[15,16]$ for more details.

In [17], the computational complexity of the supremal controllable sublanguage of a specification language $K$ with respect to the plant language $L$ with $n$ and $m$ states in their minimal generator representations, respectively, is shown (for prefix-closed languages) to be $O(m n)$. The computational complexity of the supremal controllable and normal sublanguage is $O\left(2^{m n}\right)$, see [5]. We denote the number of states of minimal generators for $L\left(G_{1}\right), L\left(G_{2}\right)$, and $L\left(G_{k}\right)$ by $m_{1}, m_{2}$, and $m_{k}$, respectively. As the specification language $K$ is conditionally decomposable, i.e., $K=$ $P_{1+k}(K)\left\|P_{2+k}(K)\right\| P_{k}(K)$, we denote the number of states of minimal generators for $P_{1+k}(K), P_{2+k}(K)$, and $P_{k}(K)$ by $n_{1}$, $n_{2}$, and $n_{k}$, respectively. Then, in the worst case, $m=O\left(m_{1} m_{2} m_{k}\right)$ and $n=O\left(n_{1} n_{2} n_{k}\right)$. The computational complexity of $\sup \mathrm{C}_{k}$, sup $\mathrm{C}_{1+k}$, and $\sup \mathrm{C}_{2+k}$ (see [8]) gives the formula $O\left(m_{k} n_{k}+m_{1} n_{1} m_{k} n_{k}+m_{2} n_{2} m_{k} n_{k}\right)$, which is better than $O(m n)=O\left(m_{1} m_{2} m_{k} n_{1} n_{2} n_{k}\right)$ of the monolithic case. The situation is more complicated for supremal controllable and normal sublanguages. In this case, the computational complexity of $\sup \mathrm{CN}_{k}$, $\sup \mathrm{CN}_{1+k}$, and $\sup \mathrm{CN}_{2+k}$ gives the formula $O\left(2^{m_{k} n_{k}}+2^{m_{1} n_{1} 2^{m_{k} n_{k}}}+2^{m_{2} n_{2} 2^{m_{k} n_{k}}}\right)$, which is better than $O\left(2^{m_{1} m_{2} m_{k} n_{1} n_{2} n_{k}}\right)$ of the monolithic case if $m_{i} n_{i}>\frac{2^{m_{k} n_{k}}}{m_{k} n_{k}}$, for $i=1,2$, i.e., if the coordinator is significantly smaller than the subsystems. As the coordinator (and its event set) can be chosen to be minimal, there is a possibility to choose the coordinator so that it, in addition, satisfies the condition that the number of states of the minimal generator of $\sup \mathrm{CN}_{k}$ is in $O\left(m_{k} n_{k}\right)$ or even in $O\left(\min \left\{m_{k}, n_{k}\right\}\right)$. However, this question requires further investigation.

In addition to the procedure for computation of $\sup \mathrm{cCN}$ in a distributed way, another consequence is of interest. Namely, under the conditions of Theorem 20, sup cCN is conditionally decomposable, cf. Lemma 23.

Lemma 23 ([8, 9]). A language $M \subseteq E^{*}$ is conditionally decomposable with respect to event sets $E_{1} \cup E_{2} \cup E_{k}=E$ if and only if there exist languages $M_{i} \subseteq E_{i}^{*}$, for $i=1,2, k$, such that $M=M_{1}\left\|M_{2}\right\| M_{k}$.

Even more, this implies that the supremal conditionally controllable and conditionally normal sublanguage is controllable and normal with respect to the global plant as we show below and, consequently, the supremal conditionally controllable and conditionally normal sublanguage is included in the global supremal controllable and normal sublanguage. This is because the language synthesized using our coordination architecture is more restrictive than the language synthesized using the supervisory control for the global plant.

Theorem 24. In the setting of Theorem $20, \sup \operatorname{cCN}\left(K, L,\left(E_{k, u}, E_{1+k, u}, E_{2+k, u}\right),\left(Q_{k}, Q_{1+k}, Q_{2+k}\right)\right)$ is controllable with respect to $L=L_{1}\left\|L_{2}\right\| L_{k}$ and $E_{u}$, and normal with respect to $L$ and $Q: E^{*} \rightarrow E_{o}^{*}$, where $E=E_{1+k} \cup E_{2+k}$.

Proof. Let $\operatorname{supcCN}=\operatorname{supcCN}\left(K, L,\left(E_{k, u}, E_{1+k, u}, E_{2+k, u}\right),\left(Q_{k}, Q_{1+k}, Q_{2+k}\right)\right)$. Controllability of sup cCN is shown in [8], thus we only prove normality here and refer the reader to [8]. Note that according to Theorem 20, there exist $\sup \mathrm{CN}_{k} \subseteq E_{k}^{*}$, sup $\mathrm{CN}_{1+k} \subseteq E_{1+k}^{*}$, and $\sup \mathrm{CN}_{2+k} \subseteq E_{2+k}^{*}$ so that $\operatorname{supcCN}=\sup \mathrm{CN}_{k}\left\|\sup \mathrm{CN}_{1+k}\right\| \sup \mathrm{CN}_{2+k}$. In addition, the following three properties hold: (1) $\sup \mathrm{CN}_{k}$ is normal with respect to $L_{k}$ and $Q_{k}$, (2) $\sup \mathrm{CN}_{1+k}$ is normal with respect to $L_{1} \| \sup \mathrm{CN}_{k}$ and $Q_{1+k}$, and (3) sup $\mathrm{CN}_{2+k}$ is normal with respect to $L_{2} \| \sup \mathrm{CN}_{k}$ and $Q_{2+k}$. Then, 
using Lemma 17,

$$
\begin{aligned}
& Q^{-1} Q(\sup c \mathrm{CN}) \cap L \| \sup \mathrm{CN}_{k} \\
&=Q^{-1} Q\left(\sup \mathrm{CN}_{k}\left\|\sup \mathrm{CN}_{1+k}\right\| \sup \mathrm{CN}_{2+k}\right) \cap L \| \sup \mathrm{CN}_{k} \\
&=Q^{-1} Q\left(P_{k}^{-1}\left(\sup \mathrm{CN}_{k}\right) \cap P_{1+k}^{-1}\left(\sup \mathrm{CN}_{1+k}\right) \cap P_{2+k}^{-1}\left(\sup \mathrm{CN}_{2+k}\right)\right) \cap L \| \sup \mathrm{CN}_{k} \\
& \subseteq Q^{-1} Q P_{k}^{-1}\left(\sup \mathrm{CN}_{k}\right) \cap Q^{-1} Q P_{1+k}^{-1}\left(\sup \mathrm{CN}_{1+k}\right) \cap Q^{-1} Q P_{2+k}^{-1}\left(\sup \mathrm{CN}_{2+k}\right) \cap L \| \sup \mathrm{CN}_{k} \\
& \subseteq P_{k}^{-1} Q_{k}^{-1} Q_{k}\left(\sup \mathrm{CN}_{k}\right) \cap P_{1+k}^{-1} Q_{1+k}^{-1} Q_{1+k}\left(\sup \mathrm{CN}_{1+k}\right) \cap P_{2+k}^{-1} Q_{2+k}^{-1} Q_{2+k}\left(\sup \mathrm{CN}_{2+k}\right) \cap L_{1}\left\|L_{2}\right\| L_{k} \| \sup \mathrm{CN}_{k} \\
&= P_{k}^{-1}\left(Q_{k}^{-1} Q_{k}\left(\sup \mathrm{CN}_{k}\right) \cap L_{k}\right) \cap P_{1+k}^{-1}\left(Q_{1+k}^{-1} Q_{1+k}\left(\sup \mathrm{CN}_{1+k}\right) \cap L_{1} \| \sup \mathrm{CN}_{k}\right) \\
& \cap P_{2+k}^{-1}\left(Q_{2+k}^{-1} Q_{2+k}\left(\sup \mathrm{CN}_{2+k}\right) \cap L_{2} \| \sup \mathrm{CN}_{k}\right) \\
&= \sup \mathrm{CN} .
\end{aligned}
$$

Thus, sup cCN is normal with respect to $L \| \sup \mathrm{CN}_{k}$ and $Q$. Analogously, we can prove that $L \| \sup \mathrm{CN}_{k}$ is normal with respect to $L \| L_{k}=L$ and $Q$. Finally, by the transitivity of normality for prefix-closed languages (Lemma 16) we obtain that sup cCN is normal with respect to $L$ and $Q$, which was to be shown.

Theorem 24 demonstrates that the result of our approach is controllable and normal with respect to $L, E_{u}$, and $Q$. To complete this study, we show that if some additional conditions are also satisfied, then the constructed supremal conditionally controllable and conditionally normal sublanguage is optimal.

Theorem 25. Consider the setting of Theorem 20. If, in addition, $L_{k} \subseteq P_{k}(L)$ and $P_{i+k}$ is OCC for the language $P_{i+k}^{-1}\left(L_{i} \| L_{k}\right)$, for $i=1,2$, then

$$
\operatorname{supcCN}\left(K, L,\left(E_{k, u}, E_{1+k, u}, E_{2+k, u}\right),\left(Q_{k}, Q_{1+k}, Q_{2+k}\right)\right)=\sup \operatorname{CN}\left(K, L, E_{u}, Q\right)
$$

if and only if

- $P_{k}\left(Q^{-1} Q(\sup \mathrm{CN}) \cap L\right)=P_{k} Q^{-1} Q(\sup \mathrm{CN}) \cap L_{k}$ and

- $P_{i+k}\left(Q^{-1} Q(\sup \mathrm{CN}) \cap L_{1}\left\|L_{2}\right\| P_{k}(\sup \mathrm{CN})\right)=P_{i+k} Q^{-1} Q(\sup \mathrm{CN}) \cap P_{i+k}\left(L_{1}\left\|L_{2}\right\| P_{k}(\sup \mathrm{CN})\right)$, for $i=1,2$,

where $\sup \mathrm{CN}=\sup \mathrm{CN}\left(K, L, E_{u}, Q\right)$.

Proof. First, note that the case considering controllability is proven in [8], i.e., $\sup \mathrm{cC}\left(K, L,\left(E_{k, u}, E_{1+k, u}, E_{2+k, u}\right)\right)=$ $\sup \mathrm{C}\left(K, L, E_{u}\right)$. Moreover, $\sup \operatorname{cCN}\left(K, L,\left(E_{k, u}, E_{1+k, u}, E_{2+k, u}\right),\left(Q_{k}, Q_{1+k}, Q_{2+k}\right)\right) \subseteq \sup \operatorname{CN}\left(K, L, E_{u}, Q\right)$ is proven in Theorem 24. Thus, it remains to show that sup CN is conditionally normal if and only if $P_{k}\left(Q^{-1} Q(\sup \mathrm{CN}) \cap L\right)=$ $P_{k} Q^{-1} Q(\sup \mathrm{CN}) \cap L_{k}$ and $P_{i+k}\left(Q^{-1} Q(\sup \mathrm{CN}) \cap L_{1}\left\|L_{2}\right\| P_{k}(\sup \mathrm{CN})\right)=P_{i+k} Q^{-1} Q(\sup \mathrm{CN}) \cap P_{1+k}\left(L_{1}\left\|L_{2}\right\| P_{k}(\sup \mathrm{CN})\right)$, for $i=1,2$.

The assumption $L_{k} \subseteq P_{k}(L)$ implies that $L_{k}=P_{k}(L)$ because $P_{k}(L) \subseteq L_{k}$ always holds. Moreover, for any $A \subseteq E^{*}$, $Q_{k}^{-1} Q_{k} P_{k}(A)=P_{k} Q^{-1} Q(A)$. This can be proven as follows. Let $x \in Q_{k}^{-1} Q_{k} P_{k}(A)$, then there exists $z \in A$ such that $Q_{k}(x)=Q_{k} P_{k}(z), P_{k}(z)=y$, and $Q(z)=w$, for some $y \in E_{k}^{*}$ and $w \in E_{o}^{*}$. Assume $Q_{k}(x)=Q_{k}(y)=v=$ $\sigma_{0} \sigma_{1} \ldots \sigma_{n}$, for $\sigma_{i} \in E_{k, o}$, or $v=\varepsilon$. Then, $x=u_{0} \sigma_{0} u_{1} \sigma_{1} \ldots u_{n} \sigma_{n} u_{n+1}$, for some $u_{i} \in\left(E_{k} \backslash E_{o}\right)^{*}$. As $Q_{k} P_{k}(z)=$ $P_{k}^{\prime} Q(z)=v$, where $P_{k}^{\prime}$ denotes the restriction of $P_{k}$ to $E_{o}^{*}, w=w_{0} \sigma_{0} w_{1} \sigma_{1} \ldots w_{n} \sigma_{n} w_{n+1}$, for $w_{i} \in\left(E_{o} \backslash E_{k}\right)^{*}$. Set $z^{\prime}=u_{0} w_{0} \sigma_{0} u_{1} w_{1} \sigma_{1} \ldots u_{n} w_{n} \sigma_{n} u_{n+1} w_{n+1}$. Then, $P_{k}\left(z^{\prime}\right)=x$ and $Q\left(z^{\prime}\right)=w$, which implies that $z^{\prime} \in Q^{-1} Q(z)$. Thus, $x \in P_{k} Q^{-1} Q(A)$. On the other hand, $x \in P_{k} Q^{-1} Q(A)$ implies that there exists $y \in Q^{-1} Q(A)$ such that $P_{k}(y)=x$, and that there is $z \in A$ such that $Q(y)=Q(z)$. Thus, considering the image of $z$ under $Q_{k} \circ P_{k}$, we obtain that $Q_{k} P_{k}(z)=$ $P_{k}^{\prime} Q(z)=P_{k}^{\prime} Q(y)=Q_{k} P_{k}(y)=Q_{k}(x)$, which implies that $x \in Q_{k}^{-1} Q_{k} P_{k}(A)$. Thus, $Q_{k}^{-1} Q_{k} P_{k}(A)=P_{k} Q^{-1} Q(A)$ is shown.

Based on these observations and the normality of $\sup \mathrm{CN}$, we obtain that the natural projection $P_{k}(\sup \mathrm{CN})=$ $P_{k}\left(Q^{-1} Q(\sup \mathrm{CN}) \cap L\right) \subseteq P_{k} Q^{-1} Q(\sup \mathrm{CN}) \cap P_{k}(L)=P_{k} Q^{-1} Q(\sup \mathrm{CN}) \cap L_{k}=Q_{k}^{-1} Q_{k} P_{k}(\sup \mathrm{CN}) \cap L_{k}$. It follows that $P_{k}(\sup \mathrm{CN})$ is normal if and only if $P_{k}\left(Q^{-1} Q(\sup \mathrm{CN}) \cap L\right)=P_{k} Q^{-1} Q(\sup \mathrm{CN}) \cap L_{k}$. Now, we show that the language $P_{1+k}(\sup \mathrm{CN})$ is normal with respect to $L_{1}\left\|P_{k}(\sup \mathrm{CN})\right\| P_{k}^{2+k}\left(L_{2} \| P_{k}(\sup \mathrm{CN})\right)$ and $Q_{1+k}$ if and only if the 
assumption is satisfied. The case of the language $P_{2+k}(\sup C N)$ is proven analogously. First, as $P_{k}(\sup \mathrm{CN}) \subseteq L_{k}$, we obtain that $Q^{-1} Q(\sup \mathrm{CN}) \cap L_{1}\left\|L_{2}\right\| P_{k}(\sup \mathrm{CN})=Q^{-1} Q(\sup \mathrm{CN}) \cap P_{1}^{-1}\left(L_{1}\right) \cap P_{2}^{-1}\left(L_{2}\right) \cap P_{k}^{-1}\left(L_{k}\right) \cap P_{k}^{-1} P_{k}(\sup \mathrm{CN})=$ $Q^{-1} Q(\sup \mathrm{CN}) \cap L_{1}\left\|L_{2}\right\| L_{k} \cap P_{k}^{-1} P_{k}(\sup \mathrm{CN})=\sup \mathrm{CN} \cap P_{k}^{-1} P_{k}(\sup \mathrm{CN})=\sup \mathrm{CN}$, i.e., sup $\mathrm{CN}$ is normal with respect to $L_{1}\left\|L_{2}\right\| P_{k}(\sup \mathrm{CN})$ and $Q$. Thus,

$$
\begin{aligned}
P_{1+k}(\sup \mathrm{CN})= & P_{1+k}\left(Q^{-1} Q(\sup \mathrm{CN}) \cap L_{1}\left\|L_{2}\right\| P_{k}(\sup \mathrm{CN})\right) \\
& \subseteq P_{1+k} Q^{-1} Q(\sup \mathrm{CN}) \cap P_{1+k}\left(L_{1}\left\|L_{2}\right\| P_{k}(\sup \mathrm{CN})\right) \\
& =Q_{1+k}^{-1} Q_{1+k} P_{1+k}(\sup \mathrm{CN}) \cap P_{1+k}\left(L_{1}\left\|L_{2}\right\| P_{k}(\sup \mathrm{CN})\right), \quad \text { by an analogous argument as above, } \\
& =Q_{1+k}^{-1} Q_{1+k} P_{1+k}(\sup \mathrm{CN}) \cap L_{1}\left\|P_{k}(\sup \mathrm{CN})\right\| P_{2 \cap k}^{2}\left(L_{2}\right), \quad \text { by Lemma } 4, \\
& =Q_{1+k}^{-1} Q_{1+k} P_{1+k}(\sup \mathrm{CN}) \cap L_{1}\left\|P_{k}(\sup \mathrm{CN})\right\| P_{2 \cap k}^{2}\left(L_{2}\right) \| P_{k}(\sup \mathrm{CN}) \\
& =Q_{1+k}^{-1} Q_{1+k} P_{1+k}(\sup \mathrm{CN}) \cap L_{1}\left\|P_{k}(\sup \mathrm{CN})\right\| P_{k}^{2+k}\left(L_{2} \| P_{k}(\sup \mathrm{CN})\right),
\end{aligned}
$$

which implies that the language $P_{1+k}(\sup \mathrm{CN})$ is normal with respect to $L_{1}\left\|P_{k}(\sup \mathrm{CN})\right\| P_{k}^{2+k}\left(L_{2} \| P_{k}(\sup \mathrm{CN})\right)$ and $Q_{1+k}$ if and only if the projection $P_{1+k}$ distributes over the languages $Q^{-1} Q(\sup \mathrm{CN})$ and $L_{1}\left\|L_{2}\right\| P_{k}(\sup \mathrm{CN})$. Hence, the proof is complete.

Note that to verify this condition, we need to compute the plant language $L$. However, this language is not computed during the computational process because of the complexity reasons. Thus, it is an open problem how to verify the optimality of the computed result based only on the local languages $L_{1}, L_{2}$, and $L_{k}$.

\subsection{An example}

In this section, we demonstrate our approach on an example. As controllability is demonstrated on an example presented in [8], we consider only the case of conditional normality here.

Let $G=G_{1} \| G_{2}$ be a plant defined over an event set $E=E_{1} \cup E_{2}=\left\{a_{1}, c, t, t_{1}\right\} \cup\left\{a_{2}, c, t, t_{2}\right\}=\left\{a_{1}, a_{2}, c, t, t_{1}, t_{2}\right\}$ as a synchronous composition of two systems $G_{1}$ and $G_{2}$ defined as shown in Figure 2, where the set of unobservable events is $E_{u o}=\left\{t, t_{1}, t_{2}\right\}$. The behaviors of these systems are $L\left(G_{1}\right)=\overline{\left\{t_{1} c, a_{1} t\right\}}, L\left(G_{2}\right)=\overline{\left\{t_{2} c, a_{2} t\right\}}$, and $L(G)=$

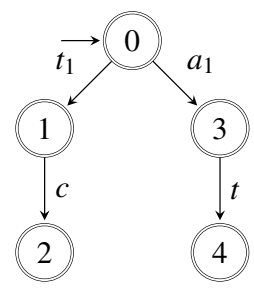

(a) Generator $G_{1}$.

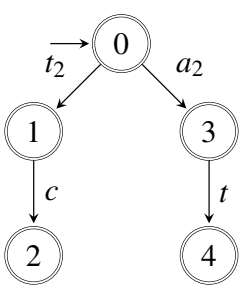

(b) Generator $G_{2}$.

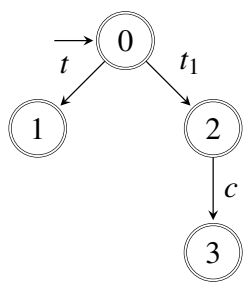

(c) Coordinator.

Figure 2: Generators $G_{1}, G_{2}$, and the coordinator.

$\overline{\left\{a_{1} a_{2} t, a_{2} a_{1} t, a_{1} t_{2}, a_{2} t_{1}, t_{1} t_{2} c, t_{2} t_{1} c, t_{1} a_{2}, t_{2} a_{1}\right\}}$. The specification $K=\overline{\left\{t_{1} t_{2} c, t_{1} a_{2}, a_{2} t_{1}, a_{2} a_{1} t, a_{1} a_{2} t, a_{1} t_{2}, t_{2} a_{1}, t_{2} t_{1}\right\}}$ is define by the generator shown in Figure 3.

Now, we need to find a coordinator $G_{k}$; specifically, its event set $E_{k}$. Note that, by the definition, $E_{k}$ has to contain both shared events $c$ and $t$. In addition, to ensure that the specification language $K$ is conditionally decomposable, at least one of $t_{1}$ and $t_{2}$ has to be added to $E_{k}$. Assume $t_{1}$ is added, i.e., $E_{k}=\left\{c, t, t_{1}\right\}$. Thus, $K$ is conditionally decomposable.

Moreover, as we consider only prefix-closed languages in this paper, and the choice of a coordinator plays a role in solving blocking issues, we choose the coordinator so that its behavior $L_{k}=L\left(G_{k}\right)$ does not change the original system when composed together, i.e., $L\left(G_{1} \| G_{2}\right) \| L_{k}=L\left(G_{1} \| G_{2}\right)$ is satisfied, see Figure 2(c). In fact, due to the absence of blocking issues, the important aspect in the choice of the coordinator is the choice of its alphabet, $E_{k}$, so that OCC and observer properties are satisfied together with the technical condition on normality.

Our choice is thus $L_{k}=L\left(P_{k}^{1}\left(G_{1}\right) \| P_{k}^{2}\left(G_{2}\right)\right)$, which means that $L_{k}=\overline{\left\{t, t_{1} c\right\}}$. The projections of $K$ are then the following languages $P_{k}(K)=\overline{\left\{t, t_{1} c\right\}}, P_{1+k}(K)=\overline{\left\{a_{1} t, t_{1} c\right\}}$, and $P_{2+k}(K)=\overline{\left\{t_{2} t_{1}, a_{2} t, a_{2} t_{1}, t_{1} a_{2}, t_{1} t_{2} c\right\}}$. We can compute 


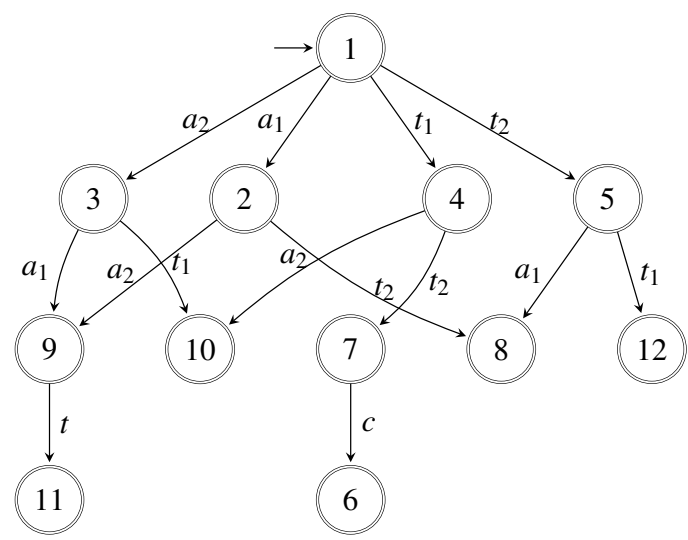

Figure 3: Generator for the specification language $K$.

the languages $\sup \mathrm{N}_{k}=\overline{\left\{t, t_{1} c\right\}}$, sup $\mathrm{N}_{1+k}=\overline{\left\{t_{1} c, a_{1} t\right\}}$, sup $\mathrm{N}_{2+k}=\overline{\left\{t_{2} t_{1}, t_{1} t_{2}, t_{1} a_{2}, a_{2} t_{1}, a_{2} t\right\}}$, as defined in Corollary 22, where their composition

$$
\sup \mathrm{N}_{k}\left\|\sup \mathrm{N}_{1+k}\right\| \sup \mathrm{N}_{2+k}=\overline{\left\{t_{2} t_{1}, t_{2} a_{1}, a_{1} t_{2}, a_{1} a_{2} t, t_{1} t_{2}, t_{1} a_{2}, a_{2} a_{1} t, a_{2} t_{1}\right\}}
$$

is the supremal conditionally normal sublanguage of $K$, which is also normal by Theorem 24 . In addition, it can be verified that the resulting language coincides with the supremal normal sublanguage of $K$ with respect to $L(G)$ and $Q$.

\section{Conclusion}

In this paper, we have investigated coordination control of modular discrete-event systems with partial observations. We have established that conditional observability together with conditional controllability form a necessary and sufficient condition for a global specification language to be exactly achievable within our coordination control that consists of local supervisors and a supervisor for the coordinator.

Similarly as observability in monolithic supervisory control with partial observations, conditional observability is not preserved by language union, hence we have studied conditional normality and supremal conditional normal sublanguages that always exist. We have shown that under quite weak assumptions supremal conditionally controllable sublanguages are conditionally decomposable and can be fairly easily computed. Sufficient conditions have been established, where a distributed computation is possible, which consists of computing the supremal conditionally controllable and conditionally normal sublanguage of a global specification language as the synchronous composition of the supremal controllable and normal sublanguages for the coordinator and those for the coordinator combined with local subsystems.

Moreover, conditions have been found under which our supremal conditionally controllable and conditionally normal sublanguage coincides with the globally optimal solution, i.e. the supremal controllable and normal sublanguage. Hence, as a consequence we have proposed an efficient computation of supremal controllable and normal sublanguage of a global specification, a very difficult problem.

For a future consideration, several extensions of our approach are left open. Let us note that the approach can be fairly easily extended to the general case of $n$ subsystems running in parallel. In fact, it is possible to introduce one central coordinator which should dispose of all events shared by at least two subsystems. Another extension to nonprefix-closed global specification languages is currently investigated. It should be noted that there is an implicit and simple form of communication between coordinator and local controllers, namely, there is a two way communication channel between the coordinator and the local supervisors such that all coordinator events are communicated between local supervisors via the coordinator. In this respect, generalizations to coordination control with more general forms of communication between coordinator and local supervisors should be investigated. Finally, it would be nice to extend the coordination control to classes of timed automata. 


\section{Acknowledgements}

The research leading to these results has received funding from the European Community's Seventh Framework Programme (FP7/2007-2013) under grant agreement no. INFSO-ICT-224498, from the Czech Academy of Sciences, Institutional Research Plan no. AV0Z10190503, and from the GAČR grants no. 103/11/0517 and 202/11/P028.

\section{References}

[1] C. G. Cassandras, S. Lafortune, Introduction to discrete event systems, Second edition, Springer, 2008

[2] J. Komenda, J. H. van Schuppen, Modular control of discrete-event systems with coalgebra, IEEE Trans. Automat. Control 53 (2008) $447-460$.

[3] J. Komenda, J. H. van Schuppen, Control of discrete-event systems with modular or distributed structure, Theoret. Comput. Sci. 388 (2007) $199-226$

[4] W. M. Wonham, Supervisory control of discrete-event systems, 2009. Lecture Notes, Department of Electrical and Computer Engineering, University of Toronto.

[5] R. D. Brandt, V. Garg, R. Kumar, F. Lin, S. I. Marcus, W. M. Wonham, Formulas for calculating supremal controllable and normal sublanguages, Systems Control Lett. 15 (1990) 111-117.

[6] B. Gaudin, H. Marchand, Supervisory control of product and hierarchical discrete event systems, Eur. J. Control 10 (2004) $131-145$.

[7] J. Komenda, J. H. van Schuppen, Coordination control of discrete event systems, in: Proc. of WODES 2008, pp. 9-15.

[8] J. Komenda, T. Masopust, J. H. van Schuppen, Supervisory control synthesis of discrete-event systems using coordination scheme, CoRR abs/1007.2707 (2010). Available on-line at http://arxiv.org/abs/1007.2707

[9] J. Komenda, T. Masopust, J. H. van Schuppen, Synthesis of safe sublanguages satisfying global specification using coordination scheme for discrete-event systems, in: Proc. of WODES 2010, pp. 436-441. Available: http://www.ifac-papersonline.net/.

[10] L. Feng, Computationally Efficient Supervisor Design for Discrete-Event Systems, Ph.D. thesis, University of Toronto, 2007.

[11] P. J. Ramadge, W. M. Wonham, Supervisory control of a class of discrete event processes, SIAM J. Control Optim. 25 (1987) 206-230.

[12] K. C. Wong, W. M. Wonham, Hierarchical control of discrete-event systems, Discrete Event Dyn. Syst. 6 (1996) $241-273$.

[13] K. Schmidt, C. Breindl, Maximally permissive hierarchical control of decentralized discrete event systems, IEEE Trans. Automat. Control $56(2011) 1-14$

[14] K. Schmidt, C. Breindl, On maximal permissiveness of hierarchical and modular supervisory control approaches for discrete event systems, in: Proc. of WODES 2008, pp. 462-467.

[15] K. C. Wong, On the complexity of projections of discrete-event systems, in: Proc. of WODES 1998, pp. 201-206.

[16] L. Feng, W. M. Wonham, On the computation of natural observers in discrete-event systems, Discrete Event Dyn. Syst. 20 (2010) 63-102.

[17] R. Kumar, V. Garg, S. I. Marcus, On controllability and normality of discrete event dynamical systems, Systems Control Lett. 17 (1991) $157-168$ 